\title{
Equivariant decomposition of polynomial vector fields
}

\author{
Fahimeh Mokhtari* \\ Jan A. Sanders ${ }^{\dagger}$ \\ Department of Mathematics, Faculty of Sciences \\ Vrije Universiteit, De Boelelaan 1081a, \\ 1081 HV Amsterdam, The Netherlands
}

September 30, 2019

\begin{abstract}
To compute the unique formal normal form of families of vector fields with nilpotent linear part, we choose a basis of the Lie algebra consisting of orbits under the linear nilpotent. This creates a new problem: to find explicit formulas for the structure constants in this new basis. These are well known in the 2D case, and recently expressions were found for the 3D case by ad hoc methods. The goal of the present paper is to formulate a systematic approach to this calculation.

We propose to do this using a rational method for the inversion of the ClebschGordan coefficients. We illustrate the method on a family of 3D vector fields and compute the unique formal normal form for the Euler family both in the 2D and 3D case.
\end{abstract}

Keywords: Invariant theory; Clebsch-Gordan coefficients; Inversion of the Clebsch-Gordan coefficients; Nilpotent singularity; Unique normal form; Euler vector field.

\section{Introduction}

Consider formal vector fields in $\mathbb{R}^{2}$ with a fixed nilpotent linear part N. In [2] the following problem was addressed: for each such vector field, determine the unique formal normal form, that is a form in which the vector field can be transformed by formal transformations such that the only transformations that are still possible without perturbing the normal form style are formal symmetries of the vector field.

This was done by constructing a new basis for the formal vector fields in such a way if $\mathrm{v}^{i}$ is a basis vector, so is $\mathrm{v}^{i+1}=\left[\mathrm{N}, \mathrm{v}^{i}\right]=\operatorname{ad}_{\mathrm{N}} \mathrm{v}^{i}$. The inversion of $\mathrm{ad}_{\mathrm{N}}$ is then a simple matter of changing the appropriate index from $i$ to $i-1$, if $i>0$. Constructing such a basis is not too difficult in lower dimensions. The problem is to compute the structure constants of the Lie algebra in this new basis. This is the equivariant decomposition of the vector fields we refer to in the title.

\footnotetext{
*Email: fahimeh.mokhtari.fm@gmail.com

$\dagger$ Email: jan.sanders.a@gmail.com
} 
In this paper, we aim to generalize this procedure from $\mathbb{R}^{2}$ to $\mathbb{R}^{n}$. Before the reader gets too excited about this, we should point out that we do not aim to solve the unique formal normal form problem for $n$-dimensional nilpotent systems. The fact that even the $2 \mathrm{D}$ results are not complete may indicate the size of such an undertaking. What we want to obtain here is a systematic procedure to compute the structure constants in a basis given by orbits of the nilpotent. This is a first step towards the determination of the unique formal normal form.

Besides from having a theoretical interest (the classification of formal vector fields under formal coordinate transformations) we should point out a practical consequence of this analysis. If one does not know what the unique formal normal form is then it is difficult to determine in a bifurcation analysis which parameters are essential and which are not (because some could be eliminated in the normalization procedure). This leads to confusion in the process of automated (numerical) bifurcation analysis.

Remark 1.1. In the sequel, we will occasionally drop the formal, but it should be clear that there are no results in this paper beyond the formal normal form.

One might ask, why go to the $3 \mathrm{D}$ case if the $2 \mathrm{D}$ case is not done yet? To give at least a partial response to this question we analyze a $2 \mathrm{D}$ subcase, the Euler vector fields, that was left open in op. cit., in Section 5.1. We will see that the 3D result is very similar to the $2 \mathrm{D}$ result. This is a phenomenon that is familiar from Classical Invariant Theory. One can expect that the 4D analysis will be much harder (but then the $5 \mathrm{D}$ case should be similar to the 4D).

The complexities of this programme are already clear in the treatment of the 3D irreducible nilpotent singularity in [6]. There the structure constants are computed using Maple computations and extrapolation, a time consuming method which does not seem to be well suited for higher dimensions. In this paper, we will formulate a procedure to compute the structure constants using two ideas:

- Use the Clebsch-Gordan coefficients to express two-tensors in terms of $\mathrm{N}$ orbits of transvectants and apply this both in the symmetrical case to products of functions and in the antisymmetrical case to Lie brackets.

- Compute the transvectants, using the fact that we know how to express the elements in ker $\operatorname{ad}_{M}$ using a Stanley basis [14, Chapter 12]. This last condition restricts at the moment the dimension $n$ to be less than 6 , at least in the irreducible case.

We hope that these techniques will enable the computation of the structure constants for the basis of $\mathrm{N}$-orbits for higher dimensions, although we realize that this will probably need some more ideas in order to be completely carried out (the difficulty being the lack of explicit formulas for the transvectants). We mention here that if one is only interested in carrying out this program for a finite jet degree, there should be no difficulty in higher dimensions; it is the infinite-dimensional character of the problem and our need for explicit formulas that is causing difficulties.

For the moment the 3D programme of computing the unique normal form with nilpotent singularity will keep us busy for a while, if the experience with the 2D case, where there are still subcases that are not completely understood, is any indication. 
The first step is to construct an $\mathfrak{s l}_{2}$-triple $\langle\mathbf{N}, \mathrm{H}, \mathrm{M}\rangle$, where $\mathrm{H}$ and $\mathrm{M}$ are also linear vector fields, using the Jacobson-Morozov Lemma (see [3, 9]). The Lie bracket relations are:

$$
\begin{aligned}
{[\mathrm{M}, \mathrm{N}] } & =\mathrm{H}, \\
{[\mathrm{H}, \mathrm{N}] } & =-2 \mathrm{~N}, \\
{[\mathrm{H}, \mathrm{M}] } & =2 \mathrm{M} .
\end{aligned}
$$

This triple is not uniquely determined, and one can make choices here that are most suitable for the given situation. This construction is possible when the linear vector fields form a reductive Lie algebra, as is the case for $\mathfrak{g l}_{n}$ and $\mathfrak{s p}_{n}$.

A formal vector field is said to be in first level normal form in the $\mathfrak{s l}_{2}$-style if its nonlinear part is contained in ker ad $\mathrm{M}_{\mathrm{M}}$. The reason for this is that the finite-dimensional representation theory of $\mathfrak{s l}_{2}$ teaches us that a finite-dimensional $\mathfrak{s l}_{2}$-module $V$ is isomorphic (as $\mathfrak{s l}_{2}$-modules, not as Lie algebras) to $\mathrm{im} \operatorname{ad}_{N} \oplus \operatorname{ker} \mathrm{ad}_{M}$ and this goes through in the infinite-dimensional formal vector field setting.

The next step is to define a basis of vector fields of a given degree $d$ by first giving a basis for the elements in ker $\operatorname{ad}_{\mathrm{M}}$ of that degree, say $\left\{\mathrm{v}_{d, 1}, \cdots, \mathrm{v}_{d, k}\right\}$ and then adding to the general basis the elements $\mathrm{v}_{d, k}^{i}=\operatorname{ad}_{\mathrm{N}}^{i} \mathrm{v}_{d, k}, k=1, \cdots, i, i=1, \cdots, \infty$. Despite the $\infty$ in this definition, this is a finite set due to the nilpotent character of $\mathrm{N}$. This way, it is easy to see what the preimage under $\mathrm{ad}_{N}$ is, but the problem is now that one does not have immediately explicit formulas for the structure constants of the Lie algebra with this basis.

\subsection{Outline}

What we want to accomplish in this paper is the following: suppose we have two polynomial vector fields, say $\operatorname{ad}_{\mathrm{N}}^{k} v$ and $\operatorname{ad}_{\mathrm{N}}^{l} w$, with $v, w \in \operatorname{ker}_{\mathrm{M}}$. Can we express the Lie bracket $\left[\operatorname{ad}_{\mathrm{M}}^{k} v, \operatorname{ad}_{\mathrm{M}}^{l} w\right]$ in a similar form, that is, as a linear combination of elements of the form $\operatorname{ad}_{\mathrm{N}}^{m_{i}} u_{i}, u_{i} \in$ ker ad $\mathrm{M}_{\mathrm{M}}$ ? Our approach will be to find a formula that expresses $\operatorname{ad}_{\mathrm{N}}^{k} v \otimes \operatorname{ad}_{\mathrm{N}}^{l} w$ as a linear combination of $\operatorname{ad}_{\mathrm{N}}^{m_{i}} u_{i}$, where the $u_{i} \in$ ker ad $\mathrm{M}_{\mathrm{M}}$ are transvectants. Transvectants were invented to be used in Classical Invariant Theory to produce from one or more given covariants a new covariant. If this sounds like something one could do forever, the results of Gordan and, somewhat more convincingly, Hilbert showed that this is in fact a finite procedure and at some point all the generating covariants are found. In our context, covariants are associated to the elements in $\mathrm{ker}_{\mathrm{ad}} \mathrm{M}$. These transvectants are linear combinations of terms of the type $\operatorname{ad}_{\mathrm{N}}^{k} v \otimes \operatorname{ad}_{\mathrm{N}}^{l} w$ and it might seem that we are running around in circles. However, the fact that the transvectants are in the kernel of $\operatorname{ad}_{M}$ gives us a way to explicitly compute them, at least in the 3D case that we will be most interested in.

We then apply these formulas to compute the unique formal normal form for general Eulerian vector fields with given nilpotent linear term in several cases.

\section{Acknowledgments}

The authors would like to express their thanks to professors Christian Kassel and Tom Koornwinder, for the email exchanges in the process of getting the inversion of the ClebschGordan coefficients right, and Bob Rink, for reading the manuscript and providing us with valuable suggestions. 


\section{Clebsch-Gordan symbols, a rational approach}

We now turn from the polynomial vector fields to a more abstract approach involving $\mathfrak{s l}_{2^{-}}$ modules. Here we write $\mathrm{N}$ for $\operatorname{ad}_{\mathrm{N}}$ to denote the $\mathfrak{s l}_{2}$-action.

The following is based on [7, Chapter XIV], to which we refer to for some of the technical details taken for granted in the the sequel. There an explicit method is given to express elements in $V_{m} \otimes V_{n}$ in terms of the $\mathrm{N}$-orbits of their transvectants (these are given in Lemma VII.7.2, loc. cit.). The only problem with this formula is that it implicitly assumes the norm of the transvectant to be 1 . Here we present a corrected version of this, restricted to the classical case $q=1$, the quantum case should be completely similar. The rational approach that is followed, see also [4], does avoid the square roots that appear in the usual quantum mechanical approach, where one works with an orthonormal base [8, 10, 16]; we are happy with orthogonality. It may also be useful in the categorification programme, see [5].

Remark 2.1. There is some historical confusion in the use of the word orthogonal in linear algebra, where the term orthogonal matrix is used for a matrix with orthonormal column vectors. When we use orthogonal we mean orthogonal and when we use orthonormal we mean orthonormal.

Let $U, V, W$ be finite-dimensional $\mathfrak{s l}_{2}$-modules. The reader may want to think of polynomial vector fields of some bounded degree or polynomials, where $\mathrm{N}$ is given, and the Jacobson-Morozov Lemma is invoked to obtain the $\mathfrak{s l}_{2}$-action. In the sequel we use $V$ to describe some properties of $\mathfrak{s l}_{2}$-modules, but this could as well be $U$ or $W$.

We know $V$ to be a direct sum of finite-dimensional H-eigenspaces $V_{m}$, with $\operatorname{dim} V_{m}=$ $m+1$ where each $V_{m}$ is generated by the $\mathrm{N}$-action on a $\mathbf{v}_{m} \in \operatorname{ker} \mathrm{M}$ with $\mathrm{Hv}_{m}=m \mathbf{v}_{m}$. We choose a basis for ker $\mathrm{M}$ consisting of eigenvectors, say

$$
\left\langle\mathrm{v}_{m_{1}}^{\{1\}}, \ldots, \mathrm{v}_{m_{1}}^{\left\{k_{m_{1}}\right\}}, \ldots, \mathrm{v}_{m_{k}}^{\{1\}}, \ldots, \mathrm{v}_{m_{k}}^{\left\{k_{m_{k}}\right\}}\right\rangle
$$

If there is not a given inner product on ker $\mathrm{M}$ we construct the standard dual basis

$$
\left\langle\mathrm{v}_{m_{1}}^{\{1 \star\}}, \ldots, \mathrm{v}_{m_{1}}^{\left\{k_{m_{1}} \star\right\}}, \ldots, \mathrm{v}_{m_{k}}^{\{1 \star\}}, \ldots, \mathrm{v}_{m_{k}}^{\left\{k_{m_{k}} \star\right\}}\right\rangle
$$

and define the standard inner product $\left(\mathrm{v}_{k}^{\{i\}}, \mathrm{v}_{l}^{\{j\}}\right)$ ker $\mathrm{M} \mid V=\mathrm{v}_{k}^{\{i \star\}}\left(\mathrm{v}_{l}^{\{j\}}\right)=\delta_{i, j} \delta_{k, l}$, with linear extension defined on all of ker $\mathrm{M}$.

Then we let for given $\mathrm{v}_{m} \in$ ker $\mathrm{M}$ with $\mathrm{H}$-eigenvalue $m$,

$$
\mathbf{v}_{m}^{i}=\mathrm{N}^{i} \mathbf{v}_{m}, \mathbf{v}_{m} \in \operatorname{ker} \mathrm{M}, \quad \mathbf{v}_{m}^{(i)}=\frac{1}{i !} \mathbf{v}_{m}^{i}, \mathrm{Nv}_{m}^{(i)}=\frac{1}{i !} \mathbf{N v}_{m}^{i}=(i+1) \mathbf{v}_{m}^{(i+1)}
$$

We denote the space spanned by $\mathbf{v}_{m}^{i}, i=0, \ldots, m$ by $V_{m}$. It follows from standard $\mathfrak{s l}_{2^{-}}$ representation theory that this way we can construct a basis for $V$.

We then define an induced inner product on $V$ by defining it on eigenvectors by

$$
\left(\mathrm{v}_{m}^{(i)}, \mathrm{w}_{n}^{(j)}\right)_{V}=\left(\begin{array}{c}
m \\
i
\end{array}\right) \delta_{i, j} \delta_{m, n}\left(\mathrm{v}_{m}, \mathrm{w}_{n}\right)_{\text {ker } \mathrm{M} \mid V}, \quad \mathbf{v}_{m}, \mathrm{w}_{n} \in \operatorname{ker} \mathrm{M} \mid V,
$$

and then by linear extension on all of $V$. The $\delta_{m, n}$ is not really necessary here, as it follows from the definition of $(\cdot, \cdot)_{\operatorname{ker} M \mid V}$, but it is used to emphasize the orthogonality of the $v_{m}^{(i)}$. 
Lemma 2.2. One has $\mathrm{N}^{\top}=\mathrm{M}$ and $\mathrm{M}^{\top}=\mathrm{N}$, where $\mathrm{N}^{\top}$ is the transpose of $\mathrm{N}$ with respect to the inner product $(\cdot, \cdot)_{V}$ :

$$
(\mathrm{Nv}, \mathrm{w})_{V}=(\mathrm{v}, \mathrm{Mw})_{V}, \quad \mathrm{v}, \mathrm{w} \in V .
$$

Proof. We compute the two sides on basis vectors:

$$
\begin{aligned}
& \left(\mathrm{Nv}_{m}^{(i)}, \mathbf{w}_{n}^{(j)}\right)_{V}=\left((i+1) \mathbf{v}_{m}^{(i+1)}, \mathbf{w}_{n}^{(j)}\right)_{V}=(i+1)\left(\begin{array}{c}
m \\
i+1
\end{array}\right) \delta_{i+1, j} \delta_{m, n}\left(\mathbf{v}_{m}, \mathbf{w}_{n}\right)_{\text {ker M }} \\
& =(m-i)\left(\begin{array}{c}
m \\
i
\end{array}\right) \delta_{i+1, j} \delta_{m, n}\left(\mathrm{v}_{m}, \mathrm{w}_{n}\right)_{\operatorname{ker} \mathrm{M}} \\
& \left(\mathrm{v}_{m}^{(i)}, \mathrm{Mw}_{n}^{(j)}\right)_{V}=\left(\mathrm{v}_{m}^{i}, \frac{1}{j !} \mathrm{MN}^{j} \mathrm{w}_{n}\right)=\left(\mathrm{v}_{m}^{(i)}, \frac{1}{(j-1) !}(m-j+1) \mathrm{N}^{j-1} \mathbf{w}_{n}\right)_{V} \\
& =(m-j+1)\left(\mathbf{v}_{m}^{(i)}, \mathbf{w}_{n}^{(j-1)}\right)_{V}=(m-i)\left(\begin{array}{c}
m \\
i
\end{array}\right) \delta_{i, j-1} \delta_{m, n}\left(\mathbf{v}_{m}, \mathbf{w}_{n}\right)_{\text {ker } \mathrm{M}} .
\end{aligned}
$$

By linear extension this holds on $V$.

Remark 2.3. This could also be formulated as: let $(\cdot, \cdot)_{V}$ be an inner product on $V$ such that $\mathrm{N}^{\top}=\mathrm{M}$. The defining property can then be readily derived.

For later use we state:

Proposition 2.4. The following binomial identity holds:

$$
\sum_{i+j=p}\left(\begin{array}{c}
m-i \\
m-p
\end{array}\right)\left(\begin{array}{l}
n-j \\
n-p
\end{array}\right)=\left(\begin{array}{c}
m+n-p+1 \\
p
\end{array}\right) .
$$

Proof. Cf. [4].

Definition 2.5. We define the $p$ th transvectant of $\mathrm{v}_{m} \otimes \mathrm{w}_{n}$,

$$
\bowtie_{m+n-2 p}: V_{m} \otimes W_{n} \rightarrow U_{m+n-2 p} \cap \operatorname{ker} \mathrm{M},
$$

as

$$
\aleph_{m+n-2 p} \mathbf{v}_{m} \otimes \mathrm{w}_{n}=\sum_{i+j=p}(-1)^{i}\left(\begin{array}{c}
p \\
i
\end{array}\right) \frac{\mathbf{v}_{m}^{(i)}}{\left(\begin{array}{c}
m \\
i
\end{array}\right)} \otimes \frac{\mathbf{w}_{n}^{(j)}}{\left(\begin{array}{c}
n \\
j
\end{array}\right)} .
$$

This definition immediately follows from the symbolic method from Classical Invariant Theory, see [12]. Alternative definitions are also in use, with multiplication constants depending on $m, n, p$, cf Remark 2.6. The notation with the $\bowtie$ is nonstandard, but it reflects the transvection process so nicely that we do not doubt Sylvester would have approved of it.

Remark 2.6. We compare the definition given in [14] with the present definition of transvectant:

$$
\begin{aligned}
(\mathrm{v}, \mathrm{w})^{(p)} & =p ! \sum_{i+j=p}(-1)^{i}\left(\begin{array}{c}
m-i \\
p-i
\end{array}\right)\left(\begin{array}{c}
n-j \\
p-j
\end{array}\right) \mathbf{v}_{m}^{i} \otimes \mathbf{w}_{n}^{j} \\
& =\frac{1}{(m-p) !(n-p) !} \sum_{i+j=p}(-1)^{i} \frac{\left(\begin{array}{c}
p \\
i
\end{array}\right)}{\left(\begin{array}{c}
m \\
i
\end{array}\right)\left(\begin{array}{c}
n \\
j
\end{array}\right)} \mathbf{v}_{m}^{(i)} \otimes \mathbf{w}_{n}^{(j)} \\
& =\frac{1}{(m-p) !(n-p) !} \bowtie_{m+n-2 p} \mathbf{v}_{m} \otimes \mathbf{w}_{n}
\end{aligned}
$$


Lemma 2.7. In order to implement the procedure given in [7] correctly, we compute

$$
\left\|\bowtie_{m+n-2 p} \mathbf{v}_{m} \otimes \mathrm{w}_{n}\right\|^{2}=\frac{\left(\begin{array}{c}
m+n-p+1 \\
p
\end{array}\right)}{\left(\begin{array}{c}
m \\
p
\end{array}\right)\left(\begin{array}{l}
n \\
p
\end{array}\right)}\left\|\mathrm{v}_{m} \otimes \mathrm{w}_{n}\right\|^{2} .
$$

Proof. One has

$$
\begin{aligned}
& \left(\bowtie_{m_{1}+n_{1}-2 p_{1}} \mathbf{v}_{m_{1}} \otimes \mathrm{w}_{n_{1}}, \bowtie_{m_{2}+n_{2}-2 p_{2}} \mathbf{v}_{m_{2}} \otimes \mathrm{w}_{n_{2}}\right)_{V \otimes W} \\
& =\sum_{i_{1}=0}^{p_{1}} \sum_{i_{2}=0}^{p_{2}}(-1)^{i_{1}+i_{2}} \frac{\left(\begin{array}{c}
p_{1} \\
i_{1}
\end{array}\right)}{\left(\begin{array}{c}
m_{1} \\
i_{1}
\end{array}\right)\left(\begin{array}{c}
n_{1} \\
p_{1}-i_{1}
\end{array}\right)} \frac{\left(\begin{array}{c}
p_{2} \\
i_{2}
\end{array}\right)}{\left(\begin{array}{c}
m_{2} \\
i_{2}
\end{array}\right)\left(\begin{array}{c}
n_{2} \\
p_{2}-i_{2}
\end{array}\right)}\left(\mathbf{v}_{m_{1}}^{\left(i_{1}\right)}, \mathbf{v}_{m_{2}}^{\left(i_{2}\right)}\right)_{V}\left(\mathbf{w}_{n_{1}}^{\left(p_{1}-i_{1}\right)}, \mathbf{w}_{n_{2}}^{\left(p_{2}-i_{2}\right)}\right)_{W} \\
& =\delta_{p_{1}, p_{2}} \delta_{m_{1}, m_{2}} \delta_{n_{1}, n_{2}}\left\|\mathbf{v}_{m}\right\|^{2}\left\|\mathbf{w}_{n}\right\|^{2} \sum_{i=0}^{p} \frac{\left(\begin{array}{c}
p \\
i
\end{array}\right)}{\left(\begin{array}{c}
m \\
i
\end{array}\right)\left(\begin{array}{c}
n \\
p-i
\end{array}\right)},
\end{aligned}
$$

and it follows that the transvectants are orthogonal and that

$$
\begin{aligned}
\left\|\bowtie_{m+n-2 p} \mathrm{v}_{m} \otimes \mathrm{w}_{n}\right\|^{2} & =\left\|\mathrm{v}_{m}\right\|^{2}\left\|\mathrm{w}_{n}\right\|^{2} \sum_{i=0}^{p} \frac{\left(\begin{array}{c}
p \\
i
\end{array}\right)^{2}}{\left(\begin{array}{c}
m \\
i
\end{array}\right)\left(\begin{array}{c}
n \\
p-i
\end{array}\right)} \\
& =\left\|\mathrm{v}_{m}\right\|^{2}\left\|\mathrm{w}_{n}\right\|^{2} \frac{p !^{2}}{m ! n !} \sum_{i=0}^{p} \frac{(m-i) !(n-p+i) !}{i !(p-i) !} .
\end{aligned}
$$

Using Proposition 2.4 we find

$$
\begin{aligned}
\sum_{i+j=p} \frac{(m-i) !(n-j) !}{i ! j !} & =\sum_{i+j=p}\left(\begin{array}{c}
m-i \\
m-p
\end{array}\right)\left(\begin{array}{c}
n-j \\
n-p
\end{array}\right) \frac{(m-p) !(p-i) !(n-p) !(p-j) !}{i ! j !} \\
& =(m-p) !(n-p) ! \sum_{i+j=p}\left(\begin{array}{c}
m-i \\
m-p
\end{array}\right)\left(\begin{array}{c}
n-j \\
n-p
\end{array}\right) \\
& =(m-p) !(n-p) !\left(\begin{array}{c}
m+n-p+1 \\
p
\end{array}\right)
\end{aligned}
$$

and it follows that

$$
\begin{aligned}
\left\|\bowtie_{m+n-2 p} \mathrm{v}_{m} \otimes \mathrm{w}_{n}\right\|^{2} & =\left\|\mathrm{v}_{m}\right\|^{2}\left\|\mathrm{w}_{n}\right\|^{2} \frac{p !^{2}}{m ! n !} \sum_{i=0}^{p} \frac{(m-i) !(n-p+i) !}{i !(p-i) !} \\
& =\frac{\left(\begin{array}{c}
m+n-p+1 \\
p
\end{array}\right)}{\left(\begin{array}{c}
m \\
p
\end{array}\right)\left(\begin{array}{l}
n \\
p
\end{array}\right)}\left\|\mathrm{v}_{m}\right\|^{2}\left\|\mathrm{w}_{n}\right\|^{2} .
\end{aligned}
$$

\section{Corollary 2.8.}

$$
\left\|\bowtie_{m+n-2 p}^{(k)} \mathbf{v}_{m} \otimes \mathbf{w}_{n}\right\|^{2}=\frac{\left(\begin{array}{c}
m+n-2 p \\
k
\end{array}\right)\left(\begin{array}{c}
m+n-p+1 \\
p
\end{array}\right)}{\left(\begin{array}{c}
m \\
p
\end{array}\right)\left(\begin{array}{l}
n \\
p
\end{array}\right)}\left\|\mathbf{v}_{m} \otimes \mathbf{w}_{n}\right\|^{2}
$$


Although we have already computed $\left\|\bowtie_{m+n-2 p}^{(k)} \mathrm{v}_{m} \otimes \mathrm{w}_{n}\right\|^{2}$, we have not computed $\aleph_{m+n-2 p}^{(k)} \mathbf{v}_{m} \otimes \mathbf{w}_{n}$ itself. We give here an explicit derivation, but mention that it also immediately follows from the symbolic method. Let $\Delta(X)=X \otimes 1+1 \otimes X$.

$$
\begin{aligned}
& \bowtie_{m+n-2 p}^{(k)} \mathrm{v}_{m} \otimes \mathrm{w}_{n}=\frac{1}{k !} \Delta^{k}(\mathrm{~N}) \bowtie_{m+n-2 p} \mathrm{v}_{m} \otimes \mathrm{w}_{n} \\
& =\frac{1}{k !} \Delta^{k}(\mathrm{~N}) \sum_{i=0}^{p}(-1)^{i} \frac{\left(\begin{array}{c}
p \\
i
\end{array}\right)}{\left(\begin{array}{c}
m \\
i
\end{array}\right)\left(\begin{array}{c}
n \\
p-i
\end{array}\right)} \mathbf{v}_{m}^{(i)} \otimes \mathbf{w}_{n}^{(p-i)} \\
& =\frac{1}{k !} \sum_{r=0}^{k} \sum_{i=0}^{p}(-1)^{i} \frac{\left(\begin{array}{c}
p \\
i
\end{array}\right)}{\left(\begin{array}{c}
m \\
i
\end{array}\right)\left(\begin{array}{c}
n \\
p-i
\end{array}\right)}\left(\begin{array}{c}
k \\
r
\end{array}\right) \frac{(i+k-r) !}{i !} \frac{(p-i+r) !}{(p-i) !} v_{m}^{(i+k-r)} \otimes \mathbf{w}_{n}^{(p-i+r)} \\
& =\sum_{r=0}^{k} \sum_{i=0}^{p}(-1)^{i} \frac{\left(\begin{array}{c}
p \\
i
\end{array}\right)}{\left(\begin{array}{c}
m \\
i
\end{array}\right)\left(\begin{array}{c}
n \\
p-i
\end{array}\right)}\left(\begin{array}{c}
i+k-r \\
k-r
\end{array}\right)\left(\begin{array}{c}
p-i+r \\
r
\end{array}\right) v_{m}^{(i+k-r)} \otimes \mathbf{w}_{n}^{(p-i+r)} \\
& =\sum_{r=0}^{k} \sum_{i=k-r}^{k-r+p}(-1)^{i-k+r} \frac{\left(\begin{array}{c}
p \\
i-k+r
\end{array}\right)}{\left(\begin{array}{c}
m \\
i-k+r
\end{array}\right)\left(\begin{array}{c}
n \\
p+k-i-r
\end{array}\right)}\left(\begin{array}{c}
i \\
k-r
\end{array}\right)\left(\begin{array}{c}
p+k-i \\
r
\end{array}\right) \mathbf{v}_{m}^{(i)} \otimes \mathbf{w}_{n}^{(p+k-i)} \\
& =\sum_{i+j=k+p} \sum_{r+q=k}(-1)^{i-k+r} \frac{\left(\begin{array}{c}
p \\
i-q
\end{array}\right)\left(\begin{array}{c}
i \\
q
\end{array}\right)\left(\begin{array}{c}
j \\
r
\end{array}\right)}{\left(\begin{array}{c}
m \\
i-q
\end{array}\right)\left(\begin{array}{c}
n \\
j-r
\end{array}\right)} \mathbf{v}_{m}^{(i)} \otimes \mathbf{w}_{n}^{(j)} \text {. }
\end{aligned}
$$

In all formulas, the summation convention is such that for a binomial expression we must have that the numbers $a$ and $b$ in $\left(\begin{array}{l}a \\ b\end{array}\right)$ are natural numbers (zero included) with $a \geq b$.

Definition 2.9. Define the Clebsch-Gordan Coefficient, also known as $3 j$-symbol , by

$$
\left(\begin{array}{ccc}
m & n & m+n-2 p \\
i & j & k
\end{array}\right)=\sum_{r+q=k}(-1)^{i-k+r} \frac{\left(\begin{array}{c}
p \\
i-q
\end{array}\right)\left(\begin{array}{l}
i \\
q
\end{array}\right)\left(\begin{array}{l}
j \\
r
\end{array}\right)}{\left(\begin{array}{c}
m \\
i-q
\end{array}\right)\left(\begin{array}{c}
n \\
j-r
\end{array}\right)}, \quad i+j=k+p,
$$

with $j_{1}=m, j_{2}=n$ and $j_{3}=n+m-2 p$ as the three $j$ 's.

Lemma 2.10. The Clebsch-Gordan Coefficients are orthogonal:

$$
\begin{gathered}
\sum\left(\begin{array}{ccc}
m_{1} & n_{1} & m_{1}+n_{1}-2 p_{1} \\
i_{1} & j_{1} & k_{1}
\end{array}\right)\left(\begin{array}{ccc}
m_{2} & n_{2} & m_{2}+n_{2}-2 p_{2} \\
i_{2} & j_{2} & k_{2}
\end{array}\right)\left(\begin{array}{c}
m_{1} \\
i_{1}
\end{array}\right)\left(\begin{array}{c}
n_{1} \\
j_{1}
\end{array}\right) \\
=\delta_{k_{1}, k_{2}} \delta_{m_{1}, m_{2}} \delta_{n_{1}, n_{2}}\left(\begin{array}{c}
m_{1}+n_{1}-2 p_{1} \\
k_{1}
\end{array}\right) \frac{\left(\begin{array}{c}
m_{1}+n_{1}-p_{1}+1 \\
p_{1}
\end{array}\right)}{\left(\begin{array}{c}
m_{1} \\
p_{1}
\end{array}\right)\left(\begin{array}{l}
n_{1} \\
p_{1}
\end{array}\right)}\left\|\mathrm{v}_{m_{1}} \otimes \mathrm{w}_{n_{1}}\right\|^{2}
\end{gathered}
$$

where the summation is over $i_{1}+j_{1}=p_{1}+k_{1}=p_{2}+k_{2}=i_{2}+j_{2}$.

Proof. We compute

$$
\begin{aligned}
& \left(\bowtie_{m_{1}+n_{1}-2 p_{1}}^{\left(k_{1}\right)} \mathrm{v}_{m_{1}} \otimes \mathrm{w}_{n_{1}}, \bowtie_{m_{2}+n_{2}-2 p_{2}}^{\left(k_{2}\right)} \mathrm{v}_{m_{2}} \otimes \mathrm{w}_{n_{2}}\right)_{V \otimes W} \\
& =\delta_{k_{1}, k_{2}}\left(\begin{array}{c}
m_{1}+n_{1}-2 p_{1} \\
k_{1}
\end{array}\right)\left(\bowtie_{m_{1}+n_{1}-2 p_{1}} \mathrm{v}_{m_{1}} \otimes \mathrm{w}_{n_{1}}, \bowtie_{m_{2}+n_{2}-2 p_{2}} \mathrm{v}_{m_{2}} \otimes \mathrm{w}_{n_{2}}\right)_{\mathrm{ker} \Delta(\mathrm{M}) \mid V \otimes W} \\
& =\delta_{k_{1}, k_{2}} \delta_{m_{1}, m_{2}} \delta_{n_{1}, n_{2}}\left(\begin{array}{c}
m_{1}+n_{1}-2 p_{1} \\
k_{1}
\end{array}\right) \frac{\left(\begin{array}{c}
m_{1}+n_{1}-p_{1}+1 \\
p_{1}
\end{array}\right)}{\left(\begin{array}{c}
m_{1} \\
p_{1}
\end{array}\right)\left(\begin{array}{c}
n_{1} \\
p_{1}
\end{array}\right)}\left\|\mathrm{v}_{m_{1}} \otimes \mathrm{w}_{n_{1}}\right\|^{2} .
\end{aligned}
$$


Theorem 2.11 (Rational Clebsch-Gordan Coefficients). The inversion formula to the definition of $\bowtie_{m+n-2 p}^{(k)} \mathrm{v}_{m} \otimes \mathrm{w}_{n}$ is

$$
\mathbf{v}_{m}^{(i)} \otimes \mathbf{w}_{n}^{(j)}=\sum_{p+k=i+j}\left(\begin{array}{ccc}
m & n & m+n-2 p \\
i & j & k
\end{array}\right) \frac{\left(\begin{array}{c}
m \\
i
\end{array}\right)\left(\begin{array}{c}
n \\
j
\end{array}\right)\left(\begin{array}{c}
m \\
p
\end{array}\right)\left(\begin{array}{l}
n \\
p
\end{array}\right)}{\left(\begin{array}{c}
m+n-2 p \\
k
\end{array}\right)\left(\begin{array}{c}
m+n-p+1 \\
p
\end{array}\right)} \aleph_{m+n-2 p}^{(k)} \mathbf{v}_{m} \otimes \mathbf{w}_{n} .
$$

Remark 2.12. We compute the special case $p=0$.

$$
\left(\begin{array}{ccc}
m & n & m+n \\
i & j & i+j
\end{array}\right)=\sum_{r+q=i+j}(-1)^{-j+r} \frac{\left(\begin{array}{c}
0 \\
i-q
\end{array}\right)\left(\begin{array}{c}
i \\
q
\end{array}\right)\left(\begin{array}{c}
j \\
r
\end{array}\right)}{\left(\begin{array}{c}
m \\
i-q
\end{array}\right)\left(\begin{array}{c}
n \\
j-r
\end{array}\right)}=1
$$

since $i-q+j-r=0$, and therefore $q=i$ and $r=j$.

Remark 2.13. Another special case is $i=0$. We find

$$
\left(\begin{array}{ccc}
m & n & m+n-2 p \\
0 & j & k
\end{array}\right)=\frac{\left(\begin{array}{l}
j \\
k
\end{array}\right)}{\left(\begin{array}{l}
n \\
p
\end{array}\right)}
$$

and it follows that

$$
\begin{aligned}
\mathbf{v}_{m}^{(0)} \otimes \mathbf{w}_{n}^{(j)} & =\sum_{p+k=j} \frac{\left(\begin{array}{c}
j \\
k
\end{array}\right)\left(\begin{array}{c}
n \\
j
\end{array}\right)\left(\begin{array}{c}
m \\
p
\end{array}\right)}{\left(\begin{array}{c}
m+n-2 p \\
k
\end{array}\right)\left(\begin{array}{c}
m+n-p+1 \\
p
\end{array}\right)} \bowtie_{m+n-2 p}^{(k)} \mathbf{v}_{m} \otimes \mathbf{w}_{n} \\
& =\sum_{p=0}^{j} \frac{\left(\begin{array}{c}
j \\
p
\end{array}\right)\left(\begin{array}{c}
n \\
j
\end{array}\right)\left(\begin{array}{c}
m \\
p
\end{array}\right)}{\left(\begin{array}{c}
m+n-2 p \\
j-p
\end{array}\right)\left(\begin{array}{c}
m+n-p+1 \\
p
\end{array}\right)} \aleph_{m+n-2 p}^{(j-p)} \mathbf{v}_{m} \otimes \mathbf{w}_{n} .
\end{aligned}
$$

Remark 2.14. This theorem, or variants of it, is well known in quantum mechanics, and in the context of quantum groups. In the context in which we plan to use it, we have no previous examples of its use.

Proof. We consider the equation

$$
A_{[r]} \mathbf{x}=\mathbf{y}
$$

where

$$
\mathbf{x}=\left(\begin{array}{c}
\mathbf{v}_{m} \otimes \mathbf{w}_{n}^{(r)} \\
\mathbf{v}_{m}^{(1)} \otimes \mathbf{w}_{n}^{(r-1)} \\
\vdots \\
\mathbf{v}_{m}^{(r-1)} \otimes \mathbf{w}_{n}^{(1)} \\
\mathbf{v}_{m}^{(r)} \otimes \mathbf{w}_{n}
\end{array}\right), \mathbf{y}=\left(\begin{array}{c}
\bowtie_{m+n}^{(r)} \mathbf{v}_{m} \otimes \mathbf{w}_{n} \\
\bowtie_{m+n-2}^{(r-1)} \mathbf{v}_{m} \otimes \mathbf{w}_{n} \\
\vdots \\
\bowtie_{m+n-2 r+2}^{(1)} \mathbf{v}_{m} \otimes \mathbf{w}_{n} \\
\aleph_{m+n-2 r} \mathbf{v}_{m} \otimes \mathbf{w}_{n}
\end{array}\right)
$$

We first make this into an orthonormal set (orthogonality is clear from the definition of the inner product) by dividing each term in $x$ and $y$ by its norm. Thus the $i+1$ st column of $A_{[r]}$ get multiplied by the $\left\|\mathbf{v}_{m}^{(i)} \otimes \mathbf{w}_{n}^{(r-i)}\right\|$ and the $p+1$ st row divided by $\left\|\bowtie_{m+n-2 p}^{(r-p)} \mathbf{v}_{m} \otimes \mathbf{w}_{n}\right\|$. The resulting matrix is then transposed and we go back to the original bases by doing the same thing again (this is because the roles of $x$ and $y$ are now switched, and therefore multiplication is switched with division). We recall that

$$
\left\|\mathrm{v}_{m}^{(i)} \otimes \mathrm{w}_{n}^{(j)}\right\|^{2}=\left(\begin{array}{c}
m \\
i
\end{array}\right)\left(\begin{array}{c}
n \\
j
\end{array}\right)\left\|\mathrm{v}_{m} \otimes \mathrm{w}_{n}\right\|^{2}
$$


and

$$
\left\|\bowtie_{m+n-2 p}^{(k)} \mathbf{v}_{m} \otimes \mathbf{w}_{n}\right\|^{2}=\frac{\left(\begin{array}{c}
m+n-2 p \\
k
\end{array}\right)\left(\begin{array}{c}
m+n-p+1 \\
p
\end{array}\right)}{\left(\begin{array}{c}
m \\
p
\end{array}\right)\left(\begin{array}{l}
n \\
p
\end{array}\right)}\left\|\mathbf{v}_{m} \otimes \mathbf{w}_{n}\right\|^{2},
$$

with $i+j=p+k=r$.

Remark 2.15. Nice as this inversion formula for the Clebsch-Gordan coefficients may look, the reader should realize that the right-hand side contains expressions that are computable, but may not always have a nice general formula. On the bright side, we notice that in the normal form computations we often need special cases with very simple $3 j$-symbols, cf. Remark 2.13. In physics problems, it would seem the finite-dimensional results are enough, but in the computation of the structure constants of the Lie algebra of polynomial vector fields with a basis consisting of $\mathrm{N}$-orbits, we need general formulas.

Remark 2.16. Although the transvectants are formulated as a map from the tensor product $V_{m} \otimes V_{n}$ to itself, in the actual application one often considers contractions. For instance, if $V_{m}$ and $V_{n}$ have polynomial elements, one can take the product of two polynomials, defining a contraction $\pi_{\circ}: V \otimes V \rightarrow V$. If the transvectant is in ker $\Delta(\mathrm{M})$, then the transvectant after this multiplication has been carried out is in ker $\mathrm{M}$, since $\mathrm{M} f g=\mathrm{M} \pi_{\circ} f \otimes g=\pi_{\circ} \Delta(\mathrm{M}) f \otimes g$. Similar reasoning can be applied when $\pi_{\wedge} X \otimes Y=[X, Y]$, due to the Jacobi identity.

\section{Product formula for the 3D-family}

Let

$\mathrm{N}:=x \frac{\partial}{\partial y}+2 y \frac{\partial}{\partial z}, \quad \mathrm{H}=2 z \frac{\partial}{\partial z}-2 x \frac{\partial}{\partial x}, \quad \mathrm{M}:=z \frac{\partial}{\partial y}+2 y \frac{\partial}{\partial x}, \quad \mathbf{E}=x \frac{\partial}{\partial x}+y \frac{\partial}{\partial y}+z \frac{\partial}{\partial z}$,

where these operators obey the relations (1.1).

Definition 3.1. Let $\mathfrak{a}$ be the linear form in $x, y, z$ that is in ker $\mathrm{M}$ and has $\mathrm{H}$-eigenvalue 2, that is $\mathfrak{a}=z$ with our choice of operators. Let $\mathfrak{b}$ be the quadratic form in ker $\mathrm{M} \cap$ ker $\mathrm{H}$, that is $\mathfrak{b}=\pi_{\circ} \bowtie_{0} \mathfrak{a} \otimes \mathfrak{a}$, which comes down to $\mathfrak{b}=x z-y^{2}$ with our choice of operators.

Theorem 3.2. The kernel of $\mathrm{M}$, acting on $\mathbb{R}[x, y, z]$, is $\mathbb{R}[\mathfrak{a}, \mathfrak{b}]$.

Proof. See [14, Section 12.6.2].

In this section, we compute the $p$ th transvectant of $\mathfrak{a}^{\mu_{1}}$ and $\mathfrak{a}^{\mu_{2}}$, that is $\aleph_{2 \mu_{1}+2 \mu_{2}-2 p}$ $\mathfrak{a}^{\mu_{1}} \otimes \mathfrak{a}^{\mu_{2}}$, where $\mu_{1}, \mu_{2} \in \mathbb{N}$.

Definition 3.3. We define, with $m_{i}=2 \mu_{i}, i=1,2, p=2 \rho$, and $l_{1}, l_{2} \in \mathbb{N}_{0}$,

$$
\begin{aligned}
\lambda_{l_{2}, \mu_{2}}^{l_{1}, \mu_{1}}(\rho) & =\left(\begin{array}{ccc}
m_{1} & m_{2} & m_{1}+m_{2}-2 p \\
l_{1} & l_{2} & l_{1}+l_{2}-p
\end{array}\right) \frac{\left(\begin{array}{c}
m_{1} \\
l_{1}
\end{array}\right)\left(\begin{array}{c}
m_{2} \\
l_{2}
\end{array}\right)}{\left(\begin{array}{c}
m_{1}+m_{2}-2 p \\
l_{1}+l_{2}-p
\end{array}\right)\left(\begin{array}{c}
m_{1}+m_{2}-p+1 \\
p
\end{array}\right)\left(\begin{array}{c}
p \\
\rho
\end{array}\right)} \\
& \times 2^{p}\left(\begin{array}{c}
\mu_{1}+\mu_{2}-\rho \\
\rho
\end{array}\right)\left(\begin{array}{c}
\mu_{1} \\
\rho
\end{array}\right)\left(\begin{array}{c}
\mu_{2} \\
\rho
\end{array}\right) \frac{\left(l_{1}\right) !\left(l_{2}\right) !}{\left(l_{1}+l_{2}-p\right) !} .
\end{aligned}
$$


Remark 3.4. Following Remark 2.13 we have the following simplified formula of (3.1) for $l_{1}=0$,

$$
\lambda_{l_{2}, \mu_{2}}^{0, \mu_{1}}(\rho)=2^{p} p ! \frac{\left(\begin{array}{c}
\mu_{1} \\
\rho
\end{array}\right)\left(\begin{array}{c}
\mu_{2} \\
\rho
\end{array}\right)\left(\begin{array}{c}
l_{2} \\
p
\end{array}\right)\left(\begin{array}{c}
m_{2}-p \\
m_{2}-l_{2}
\end{array}\right)\left(\begin{array}{c}
\mu_{1}+\mu_{2}-\rho \\
\rho
\end{array}\right)}{\left(\begin{array}{c}
p \\
\rho
\end{array}\right)\left(\begin{array}{c}
m_{1}+m_{2}-2 p \\
l_{2}-p
\end{array}\right)\left(\begin{array}{c}
m_{1}+m_{2}-p+1 \\
p
\end{array}\right)} .
$$

Theorem 3.5. The following formula holds for the product:

$$
\mathrm{N}^{l_{1}} \mathfrak{a}^{\mu_{1}} \cdot \mathrm{N}^{l_{2}} \mathfrak{a}^{\mu_{2}}=\sum_{p+k=l_{1}+l_{2}} \lambda_{l_{2}, \mu_{2}}^{l_{1}, \mu_{1}}(\rho) \mathrm{N}^{k} \mathfrak{a}^{\mu_{1}+\mu_{2}-p} \mathfrak{b}^{\rho}
$$

Proof. We know from Remark 2.16 that $\pi_{\circ} \bowtie_{2 \mu_{1}+2 \mu_{2}-2 p} \mathfrak{a}^{\mu_{1}} \otimes \mathfrak{a}^{\mu_{2}}$ is in the kernel of M after contraction of the tensor product (since a tensor stays in the kernel if its arguments are switched and thus the symmetrized tensor is also in the kernel) and is therefore, a sum of monomials $\mathfrak{a}^{Q} \mathfrak{b}^{R}$ as follows from Theorem 3.2. Let $m_{i}=2 \mu_{i}, i=1,2$ be the H-eigenvalues of $\mathfrak{a}^{\mu_{i}}$.

Comparing the degrees we see that $\mu_{1}+\mu_{2}=Q+2 R$, while comparing the $\mathrm{H}$-eigenvalues we see that $m_{1}+m_{2}-2 p=2 Q$. Thus we have $Q=\mu_{1}+\mu_{2}-p$ and $2 R=p$. This implies that $p$ is even and we put $p=2 \rho$. Then $\rho=R$ and we know that the transvectant is $c \mathfrak{a}^{\mu_{1}+\mu_{2}-2 \rho} \mathfrak{b}^{\rho}$ if $p$ is even and zero otherwise. If we evaluate $c \mathfrak{a}^{\mu_{1}+\mu_{2}-2 \rho} \mathfrak{b}^{\rho}$ for $x=0$, we find $c \mathfrak{a}^{\mu_{1}+\mu_{2}-2 \rho} \mathfrak{b}^{p} \equiv c(-1)^{\rho} \mathfrak{a}^{\mu_{1}+\mu_{2}-2 \rho} y^{2 \rho}$. Evaluating at $x=0$ means that we can compute with $\mathrm{N}_{\text {app }}=2 y \frac{\partial}{\partial z}$ since the contribution of the $x \frac{\partial}{\partial y}$-term ends up as zero anyway.

Let $\mathbf{v}_{m_{1}}=\mathfrak{a}^{\mu_{1}}$ and $\mathbf{w}_{m_{2}}=\mathfrak{a}^{\mu_{2}}$. Recall the definition of the transvectant:

$$
\bowtie_{m+n-2 p} \mathbf{v}_{m} \otimes \mathbf{w}_{n}=\sum_{i=0}^{p}(-1)^{i} \frac{\left(\begin{array}{c}
p \\
i
\end{array}\right)}{\left(\begin{array}{c}
m \\
i
\end{array}\right)\left(\begin{array}{c}
n \\
p-i
\end{array}\right)} \mathbf{v}_{m}^{(i)} \otimes \mathbf{w}_{n}^{(p-i)}
$$

Then,

$$
\begin{aligned}
\pi_{\circ} \aleph_{m_{1}+m_{2}-2 p} \mathfrak{a}^{\mu_{1}} \otimes \mathfrak{a}^{\mu_{2}} & =\pi_{\circ} \sum_{i=0}^{p}(-1)^{i} \frac{\left(\begin{array}{c}
p \\
i
\end{array}\right)}{\left(\begin{array}{c}
m_{1} \\
i
\end{array}\right)\left(\begin{array}{c}
m_{2} \\
p-i
\end{array}\right)} \mathbf{v}_{m_{1}}^{(i)} \otimes \mathbf{w}_{m_{2}}^{(p-i)} \\
& =\pi_{\circ} \sum_{i=0}^{p} \frac{\left(\begin{array}{c}
p \\
i
\end{array}\right)}{\left(\begin{array}{c}
m_{1} \\
i
\end{array}\right)\left(\begin{array}{c}
m_{2} \\
p-i
\end{array}\right)} \frac{(-1)^{i}}{i !(p-i) !} \mathbf{N}^{i} \mathfrak{a}^{\mu_{1}} \otimes \mathbf{N}^{p-i} \mathfrak{a}^{\mu_{2}} \\
& \equiv \pi_{\circ} \sum_{i=0}^{p} \frac{\left(\begin{array}{c}
p \\
i
\end{array}\right)}{\left(\begin{array}{c}
m_{1} \\
i
\end{array}\right)\left(\begin{array}{c}
m_{2} \\
p-i
\end{array}\right)} \frac{(-1)^{i}}{i !(p-i) !} \mathbf{N}_{a p p}^{i} \mathfrak{a}^{\mu_{1}} \otimes \mathbf{N}_{a p p}^{p-i} \mathfrak{a}^{\mu_{2}} \\
& =\sum_{i=0}^{p} 2^{p} \frac{\left(\begin{array}{c}
p \\
i
\end{array}\right)}{\left(\begin{array}{c}
m_{1} \\
i
\end{array}\right)\left(\begin{array}{c}
m_{2} \\
p-i
\end{array}\right)} \frac{(-1)^{i}}{i !(p-i) !} \frac{\mu_{1} !}{\left(\mu_{1}-i\right) !} \frac{\mu_{2} !}{\left(\mu_{2}-p+i\right) !} y^{p} \mathfrak{a}^{\mu_{2}+\mu_{1}-p} \\
& \equiv 2^{p} \frac{\left(\begin{array}{c}
\mu_{1}+\mu_{2}-\rho \\
\rho
\end{array}\right)\left(\begin{array}{c}
\mu_{1} \\
\rho
\end{array}\right)\left(\begin{array}{c}
\mu_{2} \\
\rho
\end{array}\right)}{\left(\begin{array}{c}
m_{1} \\
p
\end{array}\right)\left(\begin{array}{c}
m_{2} \\
p
\end{array}\right)\left(\begin{array}{c}
p \\
\rho
\end{array}\right)} \mathfrak{a}^{\mu_{1}+\mu_{2}-p} \mathfrak{b}^{\rho} .
\end{aligned}
$$

We recall the inversion formula from Theorem 2.11:

$$
\mathbf{v}_{m}^{(i)} \otimes \mathbf{w}_{n}^{(j)}=\sum_{p+k=i+j}\left(\begin{array}{ccc}
m & n & m+n-2 p \\
i & j & k
\end{array}\right) \frac{\left(\begin{array}{c}
m \\
i
\end{array}\right)\left(\begin{array}{c}
n \\
j
\end{array}\right)\left(\begin{array}{c}
m \\
p
\end{array}\right)\left(\begin{array}{c}
n \\
p
\end{array}\right)}{\left(\begin{array}{c}
m+n-2 p \\
k
\end{array}\right)\left(\begin{array}{c}
m+n-p+1 \\
p
\end{array}\right)} \aleph_{m+n-2 p}^{(k)} \mathbf{v}_{m} \otimes \mathbf{w}_{n} .
$$


This gives us a formula for $\mathrm{N}^{l_{1}} \mathfrak{a}^{\mu_{1}} \cdot \mathrm{N}^{l_{2}} \mathfrak{a}^{\mu_{2}}$, with $\mathbf{v}_{m_{i}}=\mathfrak{a}^{\mu_{i}}$ for $i=1,2$ :

$$
\begin{aligned}
& \frac{1}{l_{1} ! l_{2} !} \mathrm{N}^{l_{1}} \mathfrak{a}^{\mu_{1}} \cdot \mathrm{N}^{l_{2}} \mathfrak{a}^{\mu_{2}} \\
= & \pi_{\circ} \sum_{p+k=l_{1}+l_{2}}\left(\begin{array}{ccc}
m_{1} & m_{2} & m_{1}+m_{2}-2 p \\
l_{1} & l_{2} & k
\end{array}\right) \frac{\left(\begin{array}{c}
m_{1} \\
l_{1}
\end{array}\right)\left(\begin{array}{c}
m_{2} \\
l_{2}
\end{array}\right)\left(\begin{array}{c}
m_{1} \\
p
\end{array}\right)\left(\begin{array}{c}
m_{2} \\
p
\end{array}\right)}{\left(\begin{array}{c}
m_{1}+m_{2}-2 p \\
k
\end{array}\right)\left(\begin{array}{c}
m_{1}+m_{2}-p+1 \\
p
\end{array}\right)} \aleph_{m_{1}+m_{2}-2 p}^{(k)} \mathbf{v}_{m_{1}} \otimes \mathbf{v}_{m_{2}} \\
= & \sum_{p+k=l_{1}+l_{2}}\left(\begin{array}{ccc}
m_{1} & m_{2} & m_{1}+m_{2}-2 p \\
l_{1} & l_{2} & k
\end{array}\right) \frac{\left(\begin{array}{c}
m_{1} \\
l_{1}
\end{array}\right)\left(\begin{array}{c}
m_{2} \\
l_{2}
\end{array}\right)}{\left(\begin{array}{c}
m_{1}+m_{2}-2 p \\
k
\end{array}\right)\left(\begin{array}{c}
m_{1}+m_{2}-p+1 \\
2 \rho
\end{array}\right)\left(\begin{array}{c}
p \\
\rho
\end{array}\right)} \\
\times & 2^{p}\left(\begin{array}{c}
\mu_{1}+\mu_{2}-\rho \\
\rho
\end{array}\right)\left(\begin{array}{c}
\mu_{1} \\
\rho
\end{array}\right)\left(\begin{array}{c}
\mu_{2} \\
\rho
\end{array}\right) \frac{1}{k !} \mathrm{N}^{k} \mathfrak{a}^{\mu_{1}+\mu_{2}-p} \mathfrak{b}^{\rho} .
\end{aligned}
$$

Using Definition 3.3 we have

$$
\mathrm{N}^{l_{1}} \mathfrak{a}^{\mu_{1}} \cdot \mathrm{N}^{l_{2}} \mathfrak{a}^{\mu_{2}}=\sum_{p+k=l_{1}+l_{2}} \lambda_{l_{2}, \mu_{2}}^{l_{1}, \mu_{1}}(\rho) \mathbf{N}^{k} \mathfrak{a}^{\mu_{1}+\mu_{2}-p} \mathfrak{b}^{\rho}
$$

and this is the desired result.

Remark 3.6. This reduces, using Remark 2.12, for $\rho=0$ to

$$
\lambda_{l_{2}, \mu_{2}}^{l_{1}, \mu_{1}}(0)=\left(\begin{array}{ccc}
m_{1} & m_{2} & m_{1}+m_{2} \\
l_{1} & l_{2} & l_{1}+l_{2}
\end{array}\right) \frac{\left(\begin{array}{c}
m_{1} \\
l_{1}
\end{array}\right)\left(\begin{array}{c}
m_{2} \\
l_{2}
\end{array}\right)}{\left(\begin{array}{c}
m_{1}+m_{2} \\
l_{1}+l_{2}
\end{array}\right)\left(\begin{array}{c}
l_{1}+l_{2} \\
l_{1}
\end{array}\right)}=\frac{\left(\begin{array}{c}
m_{1} \\
l_{1}
\end{array}\right)\left(\begin{array}{c}
m_{2} \\
l_{2}
\end{array}\right)}{\left(\begin{array}{c}
m_{1}+m_{2} \\
l_{1}+l_{2}
\end{array}\right)\left(\begin{array}{c}
l_{1}+l_{2} \\
l_{1}
\end{array}\right)}=\frac{\left(\begin{array}{c}
m_{1}+m_{2}-l_{1}-l_{2} \\
m_{1}-l_{1}
\end{array}\right)}{\left(\begin{array}{c}
m_{1}+m_{2} \\
m_{1}
\end{array}\right)} .
$$

\section{Structure constants for the $\mathscr{A}_{2}$-family}

Definition 4.1. The 3D Euler family $\mathscr{A}_{2}$ is defined by the following (affine) vector spaces

$$
\mathscr{A}_{2}=\left\langle\mathrm{A}_{\mu, k}^{l}\right\rangle_{0 \leqslant l \leqslant 2 \mu, \mu, k \in \mathbb{N}_{0}}, \quad \widehat{\mathscr{A}_{2}}=\mathrm{N}+\mathscr{A}_{2},
$$

where $\mathrm{A}_{\mu, k}^{l}=\operatorname{ad}_{\mathrm{N}}^{l} \mathfrak{a}^{\mu} \mathfrak{b}^{k} \mathrm{E}=\left(\mathrm{N}^{l} \mathfrak{a}^{\mu}\right) \mathfrak{b}^{k} \mathrm{E}$. We observe that $\mathrm{HA}_{\mu, k}^{l}=(m-2 l) \mathrm{A}_{\mu, k}^{l}$, with $m=2 \mu$.

The aim of this part, is to derive explicit formulas for the structure constants of the $\mathscr{A}_{2}$-family.

Theorem 4.2. The following holds, with $p=2 \rho$ :

$$
\left[\mathrm{A}_{\mu_{1}, k_{1}}^{l_{1}}, \mathrm{~A}_{\mu_{2}, k_{2}}^{l_{2}}\right]=\left(\mu_{2}+2 k_{2}-\mu_{1}-2 k_{1}\right) \sum_{p+k=l_{1}+l_{2}} \lambda_{l_{2}, \mu_{2}}^{l_{1}, \mu_{1}}(\rho) \mathrm{A}_{\mu_{1}+\mu_{2}-p, k_{1}+k_{2}+\rho}^{k} .
$$

Proof. The structure constants for $\mathscr{A}_{2}$ of triple-zero singularities are (on a basis of homogeneous vector fields)

$$
[f \mathrm{E}, g \mathrm{E}]=f(\mathrm{E} g) \mathrm{E}-g(\mathrm{E} f) \mathrm{E}=\left(\delta_{0}(g)-\delta_{0}(f)\right) f g \mathrm{E},
$$

where $\delta_{0}$ is the degree. This shows that $\mathscr{A}_{2}$ is a Lie subalgebra of the $3 \mathrm{D}$ polynomial vector fields. Applying this formula and (3.3) we have

$$
\begin{aligned}
{\left[\mathrm{A}_{i_{1}, k_{1}}^{l_{1}}, \mathrm{~A}_{i_{2}, k_{2}}^{l_{2}}\right] } & =\left(\delta_{0}\left(\mathrm{~A}_{i_{2}, k_{2}}^{l_{2}}\right)-\delta_{0}\left(\mathrm{~A}_{i_{1}, k_{1}}^{l_{1}}\right)\right) \mathrm{N}^{l_{1}} \mathfrak{a}^{i_{1}} \cdot \mathrm{N}^{l_{2}} \mathfrak{a}^{i_{2}} \mathfrak{b}^{k_{1}+k_{2}} \mathrm{E} \\
& =\left(\delta_{0}\left(\mathrm{~A}_{i_{2}, k_{2}}^{l_{2}}\right)-\delta_{0}\left(\mathrm{~A}_{i_{1}, k_{1}}^{l_{1}}\right)\right) \sum_{p+k=l_{1}+l_{2}} \lambda_{l_{2}, i_{2}}^{l_{1}, i_{1}}(\rho) \mathrm{N}^{k} \mathfrak{a}^{i_{1}+i_{2}-p} \mathfrak{b}^{k_{1}+k_{2}+\rho} \mathrm{E} \\
& =\left(\delta_{0}\left(\mathrm{~A}_{i_{2}, k_{2}}^{l_{2}}\right)-\delta_{0}\left(\mathrm{~A}_{i_{1}, k_{1}}^{l_{1}}\right)\right) \sum_{p+k=l_{1}+l_{2}} \lambda_{l_{2}, i_{2}}^{l_{1}, i_{1}}(\rho) \mathrm{A}_{i_{1}+i_{2}-p, k_{1}+k_{2}+\rho}^{k} .
\end{aligned}
$$


This gives us an equivariant decomposition with explicit structure constants (where explicit is a bit restricted by the $3 j$-symbols, which are given as a finite sum).

Corollary 4.3. Notice that if we choose to work $\bmod \mathfrak{b}^{k_{1}+k_{2}+1}$, formula (3.3) reduces to

$$
\left[\mathrm{A}_{i_{1}, k_{1}}^{l_{1}}, \mathrm{~A}_{i_{2}, k_{2}}^{l_{2}}\right]=\left(\delta_{0}\left(\mathrm{~A}_{i_{2}, k_{2}}^{l_{2}}\right)-\delta_{0}\left(\mathrm{~A}_{i_{1}, k_{1}}^{l_{1}}\right)\right) \lambda_{l_{2}, i_{2}}^{l_{1}, i_{1}}(0) \mathrm{A}_{i_{1}+i_{2}, k_{1}+k_{2}}^{l_{1}+l_{2}}, \quad \bmod \mathfrak{b}^{k_{1}+k_{2}+1}
$$

This makes the formula explicit, cf. Remark 3.6.

Example 4.4. These are some examples of structure constants of the $\mathscr{A}_{2}$ Lie algebra:

$$
\begin{aligned}
{\left[\mathrm{A}_{3,0}^{2}, \mathrm{~A}_{13,0}^{14}\right]=} & \frac{325}{16182} \mathrm{~A}_{16,16,0}-\frac{208}{93} \mathrm{~A}_{14,14,1}+\frac{146578432}{23} \mathrm{~A}_{10,10,3}-\frac{7192640}{2001} \mathrm{~A}_{12,12,2} \\
{\left[\mathrm{~A}_{7,2}^{8}, \mathrm{~A}_{7,1}^{5}\right]=} & -\frac{4004}{200583} \mathrm{~A}_{12,4}^{11}-\frac{4330871193600}{46189} \mathrm{~A}_{4,8}^{3}-\frac{53760000}{5681} \mathrm{~A}_{8,6}^{7}+\frac{5268480000}{3553} \mathrm{~A}_{6,7}^{5} \\
& +\frac{312134860800}{221} \mathrm{~A}_{2,9}^{1}+\frac{13505184}{482885} \mathrm{~A}_{10,5}^{9}-\frac{1001}{4011660} \mathrm{~A}_{14,3}^{13} .
\end{aligned}
$$

\section{Normal form studies for the $\widehat{\mathscr{A}}$-family}

In this part, we intend to find the structure constants and unique normal form for the $\mathscr{A}$ family of 2D and 3D nilpotent singularities, cf. [2] and [6. Equation 4.19], were in the 3D case we apply the inversion formula of the Clebsch-Gordan coefficients. Here, these families

are called $\widehat{\mathscr{A}_{1}}$ and $\widehat{\mathscr{A}_{2}}$ respectively, see (5.4) and (4.1). Apart from technical complications in the $3 \mathrm{D}$ case, the unique normal form computation is very similar in these two cases, so it makes sense to read the $2 \mathrm{D}$ case before going to the $3 \mathrm{D}$ case.

\subsection{Unique normal for the $\widehat{\mathscr{A}_{1}}$-family}

One should note that the unicity results of this part are new for the third level normal form and do not follow from the results of [2].

The purpose of this part is to study the unique normal form of the following system

$$
\left(\begin{array}{l}
\dot{x} \\
\dot{y}
\end{array}\right)=\left(\begin{array}{ll}
0 & 0 \\
1 & 0
\end{array}\right)\left(\begin{array}{l}
x \\
y
\end{array}\right)+\sum_{m=0}^{\infty} \sum_{n=0}^{m} a_{m, n}^{(0)} x^{n} y^{m-n}\left(\begin{array}{l}
x \\
y
\end{array}\right),
$$

with $a_{0,0}^{(0)}=0$ and $a_{m, n}^{(0)}$ real constants. Let $\mathfrak{g l}_{2}$ is defined by $\mathrm{N}, \mathrm{H}, \mathrm{M}, \mathrm{E}$ by the following formulas

$$
\begin{array}{ll}
\mathbf{N}=x \frac{\partial}{\partial y}, & \mathrm{H}=y \frac{\partial}{\partial y}-x \frac{\partial}{\partial x}, \\
\mathbf{M}=y \frac{\partial}{\partial x}, & \mathbf{E}=x \frac{\partial}{\partial x}+y \frac{\partial}{\partial y} .
\end{array}
$$

Then let $\mathfrak{a}$ be the linear form in $x, y$ that is in ker $\mathrm{M}$ and has $\mathrm{H}$-eigenvalue 1. Recall from [2,11] the $\mathfrak{s l}_{2}$-representation for two dimensional vector field by

$$
\mathrm{A}_{m}^{n}:=\mathrm{N}^{n} \mathfrak{a}^{m} \mathrm{E}, \quad(0 \leq n \leq m)
$$


The following special cases are useful for the unique normal form study of (5.1):

$$
\begin{aligned}
{\left[\mathrm{M}, \mathrm{A}_{m}^{0}\right] } & =0, \\
{\left[\mathrm{~N}, \mathrm{~A}_{m}^{n}\right] } & =\mathrm{A}_{m}^{n+1}, n<m, \\
{\left[\mathrm{~N}, \mathrm{~A}_{m}^{m}\right] } & =0, \\
{\left[\mathrm{~A}_{\nu}^{0}, \mathrm{~A}_{m}^{n}\right] } & =(m-\nu) \mathrm{A}_{m+\nu}^{n} .
\end{aligned}
$$

Define

$$
\mathscr{A}_{1}=\left\langle\mathrm{A}_{i}^{l}\right\rangle_{i=1, \cdots, \infty, l=0, \cdots, i}, \quad \widehat{\mathscr{A}_{1}}=\mathrm{N}+\mathscr{A}_{1} .
$$

Using the $\mathfrak{s l}_{2}$-structure one can rewrite (5.1) in the following form

$$
\mathrm{v}^{(0)}=\mathrm{N}+\sum_{s=1}^{\infty} \sum_{l=0}^{s} a_{s, l}^{(0)} \mathrm{A}_{s}^{l} \in \widehat{\mathscr{A}_{1}}
$$

in which the $a_{s, l}^{(0)}$ are real constants.

Proposition 5.1. The first level normal form of (5.5) is given by

$$
\mathrm{v}^{(1)}=\mathrm{N}+\sum_{s=1}^{\infty} a_{s}^{(1)} \mathrm{A}_{s}^{0} \in \widehat{\mathscr{A}_{1}}
$$

where the $a_{s}^{(1)}$ are the coefficients of the first level formal normal form.

Proof. The result follows from the $\mathfrak{s l}_{2}$-style normal form applied to Equation (5.5). See Proposition 5.1] in this paper, [1,13] and [14, page 201] for more details. The transformation generator at grade $s$ can be obtained by solving the Homological Equation

$$
\operatorname{ad}_{M}\left(A_{s}^{l}-\operatorname{ad}_{N} T_{s}^{l}\right)=0 .
$$

One sees that if $l>0, \mathrm{~T}_{s}^{l}=\mathrm{A}_{s}^{l-1}$ is a solution. The terms $\mathrm{A}_{s}^{0}$ are in $\operatorname{ker}_{\mathrm{M}} \mathrm{M}$ and therefore in the chosen complement of $\mathrm{im} \mathrm{ad}_{\mathrm{M}}$ and we can take $\mathrm{T}_{0}^{l}=0$. The ease of this solution procedure is the motivation for the choice of basis.

We finish this part by giving the unique normal form of (5.5).

Define $\nu_{1}:=\min \left\{s \mid a_{s}^{(1)} \neq 0\right\}$, where the $a_{s}^{(1)}$ are the first level normal form coefficients of (5.6).

Remark 5.2. Since we are going to divide by $a_{\nu_{1}}^{(1)}$ in the process of computing the transformation, one might want to impose some more requirements on this number. One could for instance stipulate that $\left|a_{\nu_{1}}^{1}\right|>0.3$, thus avoiding big numbers in the formal transformation. The numbers that we consider too small could be seen as versal deformation parameters. In this paper, we will not complicate our results by these considerations but leave it to the reader to fill in the necessary modifications. 
By rewriting Equation (5.6) we obtain

$$
v^{(1)}:=\mathrm{N}+a_{\nu_{1}}^{(1)} \mathrm{A}_{\nu_{1}}^{0}+\sum_{s=\nu_{1}+1}^{\infty} a_{s}^{(1)} \mathrm{A}_{s}^{0} .
$$

Define the grading $\delta_{2}$ by

$$
\delta_{2}\left(Z_{k}^{l}\right):=2\left(k+\nu_{1} l\right)
$$

Hence $\delta_{2}\left(\mathrm{~A}_{\nu_{1}}^{0}\right)=\delta_{2}(\mathrm{~N})=2 \nu_{1}$. Define $\mathrm{X}^{2 \nu_{1}}=\mathrm{N}+a_{\nu_{1}}^{(1)} \mathrm{A}_{\nu_{1}}^{0}$. Now we use the kernel of $\mathrm{N}$ which is generated by $\left\{\mathrm{A}_{m}^{m} \mid m \geq 1\right\}$ to eliminate extra terms from (5.7). But first we formulate the following lemma.

Lemma 5.3. There exists a transformation $\mathrm{T}_{m}^{n}=\sum_{i=0}^{n-1} \alpha_{i} \mathrm{~A}_{m+i \nu_{1}}^{n-i-1} \in \mathscr{A}_{1}$ such that the following holds:

$$
\left[\mathrm{X}^{2 \nu_{1}}, \mathrm{~T}_{m}^{n}\right]+\mathrm{A}_{m}^{n}=\alpha_{n} \mathrm{~A}_{m+n \nu_{1}}^{0}
$$

in which

$$
\alpha_{i}=-\prod_{j=1}^{i} a_{\nu_{1}}^{(1)}\left(\delta_{0}\left(\mathrm{~A}_{\nu_{1}}^{0}\right)-\delta_{0}\left(\mathrm{~A}_{m+(j-1) \nu_{1}}^{n-j}\right)\right), \quad \text { for } 0 \leq i \leq n
$$

Proof. We intend to find the coefficient of previous vector field such that

$$
\left[\mathrm{T}_{m}^{n}, \mathrm{X}^{2 \nu_{1}}\right]+\mathrm{A}_{m}^{n} \in \operatorname{ker}\left(\operatorname{ad}_{\mathrm{M}}\right)
$$

By straightforward calculation one has

$$
\begin{aligned}
{\left[\mathrm{X}^{2 \nu_{1}}, \mathrm{~T}_{m}^{n}\right]+\mathrm{A}_{m}^{n}=} & \mathrm{A}_{m}^{n}+\left[\mathrm{N}+a_{\nu_{1}}^{(1)} \mathrm{A}_{\nu_{1}}^{0}, \sum_{i=0}^{n-1} \alpha_{i} \mathrm{~A}_{m+i \nu_{1}}^{n-i-1}\right] \\
= & \mathrm{A}_{m}^{n}+\sum_{i=0}^{n-1} \alpha_{i} \mathrm{~A}_{m+i \nu_{1}}^{n-i}+\sum_{i=0}^{n-1}\left(\delta_{0}\left(\mathrm{~A}_{m+i \nu_{1}}^{n-i-1}\right)-\delta_{0}\left(\mathrm{~A}_{\nu_{1}}^{0}\right)\right) \alpha_{i} a_{\nu_{1}}^{(1)} \mathrm{A}_{m+i \nu_{1}+\nu_{1}}^{n-i-1} \\
= & \mathrm{A}_{m}^{n}+\alpha_{0} \mathrm{~A}_{m}^{n}+\sum_{i=1}^{n-1}\left(\alpha_{i}+\left(\delta_{0}\left(\mathrm{~A}_{m+(i-1) \nu_{1}}^{n-i}\right)-\delta_{0}\left(\mathrm{~A}_{\nu_{1}}^{0}\right)\right) \alpha_{i-1} a_{\nu_{1}}^{(1)}\right) \mathrm{A}_{m+i \nu_{1}}^{n-i} \\
& +\left(\delta_{0}\left(\mathrm{~A}_{m+(n-1) \nu_{1}}^{0}\right)-\delta_{0}\left(\mathrm{~A}_{\nu_{1}}^{0}\right)\right) \alpha_{n-1} a_{\nu_{1}}^{(1)} \mathrm{A}_{m+n \nu_{1}}^{0} .
\end{aligned}
$$

Now, let $\alpha_{0}=-1$ and

$$
\alpha_{i}=\left(\delta_{0}\left(\mathrm{~A}_{\nu_{1}}^{0}\right)-\delta_{0}\left(\mathrm{~A}_{m+(i-1) \nu_{1}}^{n-i}\right)\right) a_{\nu_{1}}^{(1)} \alpha_{i-1}, \quad \text { for } 1 \leq i \leq n
$$

Finally we obtain

$$
\left[\mathrm{X}^{2 \nu_{1}}, \mathrm{~T}_{m}^{n}\right]+\mathrm{A}_{m}^{n}=\left(\delta_{0}\left(\mathrm{~A}_{m+(n-1) \nu_{1}}^{0}\right)-\delta_{0}\left(\mathrm{~A}_{\nu_{1}}^{0}\right)\right) \alpha_{n-1} a_{\nu_{1}}^{(1)} \mathrm{A}_{m+n \nu_{1}}^{0}=-\alpha_{n} \mathrm{~A}_{m+n \nu_{1}}^{0}
$$


Theorem 5.4. There exists a transformation that takes (5.1) into its second level normal form

$$
\mathrm{v}^{(2)}:=\mathrm{N}+a_{\nu_{1}}^{(1)} \mathrm{A}_{\nu_{1}}^{0}+\sum_{s=\nu_{1}+1}^{\infty} a_{s}^{(2)} \mathrm{A}_{s}^{0}
$$

where $a_{s}^{(2)}$ is zero for $s \stackrel{\left(1+\nu_{1}\right)}{=} \nu_{1}$.

Proof. As mentioned before, $\mathrm{A}_{m}^{m}$ is in ker $\operatorname{ad}_{N}$. Then,

$$
\left[\mathrm{X}^{2 \nu_{1}}, \mathrm{~A}_{m}^{m}\right]=\left(m-\nu_{1}\right) \mathrm{A}_{m+\nu_{1}}^{m} .
$$

Let us assume that $m \neq \nu_{1}$. If $m=\nu_{1}$ then $A_{\nu_{1}}^{\nu_{1}}$ generates the kernel for $\mathrm{X}^{2 \nu_{1}}$. This kernel term will be used in the last theorem of this section to provide the unique normal form. Now, by applying Lemma 5.3 we get

$$
\left[\mathrm{X}^{2 \nu_{1}}, \mathrm{~A}_{m}^{m}+\left(m-\nu_{1}\right) \mathrm{T}_{m+\nu_{1}}^{m}\right]=-\alpha_{m}\left(m-\nu_{1}\right) \mathrm{A}_{m+m \nu_{1}+\nu_{1}}^{0}
$$

where $\alpha_{m}$ is nonzero.

From (5.3) we have $\left[A_{\nu_{1}}^{0}, A_{\nu_{1}}^{\nu_{1}}\right]=0$. Then $A_{\nu_{1}}^{\nu_{1}}$ generates the kernel for $X^{2 \nu_{1}}$. Set

$$
\nu_{2}=\min \left\{s \mid a_{s}^{(2)} \neq 0\right\} .
$$

We close this section by giving the main theorem about the unique normal form of (5.5).

Theorem 5.5. There exists the transformation that takes (5.8) into its unique (third level) normal form

$$
\mathrm{v}^{(3)}:=\mathrm{N}+a_{\nu_{1}}^{(1)} \mathrm{A}_{\nu_{1}}^{0}+a_{\nu_{2}}^{(2)} \mathrm{A}_{\nu_{2}}^{0}+\sum_{s=\nu_{2}+1}^{\infty} a_{s}^{(3)} \mathrm{A}_{s}^{0}
$$

or equivalently

$$
\left(\begin{array}{l}
\dot{x} \\
\dot{y}
\end{array}\right)=\left(\begin{array}{ll}
0 & 0 \\
1 & 0
\end{array}\right)\left(\begin{array}{l}
x \\
y
\end{array}\right)+a_{\nu_{1}}^{(1)} y^{\nu_{1}}\left(\begin{array}{l}
x \\
y
\end{array}\right)+a_{\nu_{2}}^{(2)} y^{\nu_{2}}\left(\begin{array}{l}
x \\
y
\end{array}\right)+\sum_{s=\nu_{2}+1}^{\infty} a_{s}^{(3)} y^{s}\left(\begin{array}{l}
x \\
y
\end{array}\right),
$$

where $a_{s}^{(3)}$ is zero for $s \stackrel{\left(1+\nu_{1}\right)}{\equiv} \nu_{1}$ and $s \stackrel{\left(1+\nu_{1}\right)}{\equiv} \nu_{2}$.

Proof. From the structure constants one has

$$
\left[\mathrm{A}_{\mu}^{0}, \mathrm{~A}_{m}^{m}\right]=(m-\mu) A_{m+\mu}^{m}, \quad \text { with }\left\{\begin{array}{lll}
\mu=\nu_{2}, & \text { if } & m=\nu_{1}, \\
\mu=\nu_{1}, & \text { if } & m \neq \nu_{1} .
\end{array}\right.
$$

By applying Lemma 5.3 one obtains

$$
\left[\mathrm{A}_{\mu}^{0}, \mathrm{~A}_{m}^{m}\right]+\left[\mathrm{X}^{2 \nu_{1}},(m-\mu) \mathrm{T}_{m+\mu}^{m}\right]=-(m-\mu) \alpha_{m} \mathrm{~A}_{m+\mu+m \nu_{1}}^{0},
$$

which is non-zero. Hence for $m=\nu_{1}$ and $\mu=\nu_{2}$ one obtains

$$
\left[\mathrm{A}_{\nu_{2}}^{0}, \mathrm{~A}_{\nu_{1}}^{\nu_{1}}\right]+\left[\mathrm{X}^{2 \nu_{1}},\left(\nu_{1}-\nu_{2}\right) \mathrm{T}_{\nu_{1}+\nu_{2}}^{\nu_{1}}\right]=\left(\nu_{2}-\nu_{1}\right) \alpha_{m} \mathrm{~A}_{\nu_{1}+\nu_{2}+\nu_{1}^{2}}^{0} .
$$

Therefore, the term $A_{\nu_{1}+\nu_{2}+\nu_{1}^{2}}^{0}$ can be eliminated from (5.8). 


\subsection{Unique normal for the $\widehat{\mathscr{A}_{2}}$-family}

In this section, we study the unique normal form for the class of equations of the form

$$
\left(\begin{array}{c}
\dot{x} \\
\dot{y} \\
\dot{z}
\end{array}\right)=\left(\begin{array}{ccc}
0 & 0 & 0 \\
1 & 0 & 0 \\
0 & 2 & 0
\end{array}\right)\left(\begin{array}{l}
x \\
y \\
z
\end{array}\right)+\sum_{k=0}^{\infty} \sum_{\mu=0}^{\infty} \sum_{l=0}^{2 \mu} a_{l, \mu, k}^{(0)} \mathrm{a}_{\mu, k}^{l}\left(\begin{array}{l}
x \\
y \\
z
\end{array}\right),
$$

where $a_{l, \mu, k}^{(0)} \in \mathbb{R}, a_{0,0,0}^{(0)}=0$ and $\mathrm{a}_{\mu, k}^{l}=\mathfrak{b}^{k} \mathrm{~N}^{l} \mathfrak{a}^{\mu}$ as defined in Section 4 or

$$
\mathrm{v}^{(0)}=\mathrm{N}+\sum_{k=0}^{\infty} \sum_{\mu=0}^{\infty} \sum_{l=0}^{2 \mu} a_{l, \mu, k}^{(0)} \mathrm{A}_{\mu, k}^{l} \in \widehat{\mathscr{A}_{2}}
$$

where $\mathrm{A}_{\mu, k}^{l}=\mathrm{a}_{\mu, k}^{l} \mathrm{E}$.

Lemma 5.6. There exists a sequence of vector fields belonging to $\mathscr{A}_{2}$ which takes (5.11) to the following first level normal form

$$
\mathrm{v}^{(1)}=\mathrm{N}+\sum_{\mu=0}^{\infty} \sum_{k=0}^{\infty} a_{\mu, k}^{(1)} \mathrm{A}_{\mu, k}^{0},
$$

where $a_{\mu, k}^{(1)}$ denotes the coefficients of vector fields in the first level normal form.

Proof. Recall that ker ad $\mathrm{M}_{\mathrm{M}}$ is $\mathfrak{s l}_{2}$-style normal form. Then, by fixing this style the proof follows from $\operatorname{ad}_{M} A_{\mu, k}^{0}=0$.

Now, define the grading function by taking $r=s+r_{s}$ and

$$
\delta_{r_{s}, s}\left(Z_{i, k}^{l}\right):=\left(r_{s}+1\right)(i+2 k)+(l-i)\left(r_{s}+2 s\right),
$$

in which $r_{s}=\min \left\{m \mid a_{m, s}^{(1)} \neq 0\right\}$. Hence,

$$
\delta_{r_{s}, s}(\mathrm{~N})=\delta_{r_{s}, s}\left(\mathrm{~A}_{r_{s}, s}^{0}\right)=r_{s}+2 s .
$$

Remark that $\delta_{0,0}\left(Z_{i, k}^{l}\right)=i+2 k$ coincides with the previous definition of $\delta_{0}$. Define,

$$
\mathrm{X}^{r_{s}+2 s}=\mathrm{N}+a_{r_{s}, s}^{(1)} \mathrm{A}_{r_{s}, s}^{0} .
$$

Before we start the study of further elimination of terms from Equation (5.13) we would like to discuss in details about the filtering approach that plays the main role in the rest of this paper.

From Theorem (4.3), it follows that the power of $\mathfrak{b}$ measures the filtering. In the process of normalization we would be able to get rid of all terms (with power of $\mathfrak{b}$ ) that generated in the next level of normal form.

Assume that in the second level normal form one wants to eliminate the terms $\mathrm{A}_{i, s}^{0}$ where $i>r_{s}$, then one does not need to take into account the terms with a higher power of $\mathfrak{b}^{s}$. Therefore, the transformation $\mathrm{T}^{i-r_{s}}$ can be obtained by solving the following

$$
\left[\mathrm{X}^{r_{s}+2 s}, \mathrm{~T}^{i-r_{s}}\right] \equiv \mathrm{A}_{i, s}^{0}
$$

where we start, fixing the degree $\delta=i+2 s$, our computation with $s=0$. With the degree fixed, the filtered computation will take only a finite number of steps. So first we eliminate $A_{\delta, 0}^{0}$, then $A_{\delta-2,1}^{0}$, until we have exhausted all terms in ker ad ${ }_{\mathrm{M}}$ with $\delta_{r_{s}, s}=\delta$. 
Theorem 5.7. There exists transformation as $\mathrm{T}_{m, k}^{n}$ such that the following holds:

$$
\left[\mathrm{T}_{m, k}^{n}, \mathrm{X}^{r_{s}+2 s}\right]+\mathrm{A}_{m, k}^{n} \equiv-\alpha_{n} \mathrm{~A}_{m+n r_{s}, k+n s}^{0} .
$$

Here we define $\alpha_{i}$ to be

$$
\alpha_{i}=-\prod_{j=1}^{i} a_{r_{s}, s}^{(1)}\left(\delta_{0}\left(\mathrm{~A}_{r_{s}, s}^{0}\right)-\delta_{0}\left(\mathrm{~A}_{m+j r_{s}-r_{s}, k+j s-s}^{n-j+1}\right)\right) \lambda_{n-j+1, m+j r_{s}-r_{s}}^{0, r_{s}}(0), \quad \text { for } 0 \leq i \leq n .
$$

Proof. Define

$$
\mathrm{T}_{m, k}^{n}=\sum_{i=0}^{n-1} \alpha_{i} \mathrm{~A}_{m+i r_{s}, k+i s}^{n-i-1}
$$

Then,

$$
\begin{aligned}
{\left[\mathrm{X}^{r_{s}+2 s}, \mathrm{~T}_{m, k}^{n}\right]+\mathrm{A}_{m, k}^{n}=} & \mathrm{A}_{m, k}^{n}+\sum_{i=0}^{n-1} \alpha_{i}\left[\mathrm{~N}, \mathrm{~A}_{m+i r_{s}, k+i s}^{n-i-1}\right]+\sum_{i=0}^{n-1} \alpha_{i}\left[a_{r_{s}, s}^{(1)} \mathrm{A}_{r_{s}, s}^{0}, \mathrm{~A}_{m+i r_{s}, k+i s}^{n-i-1}\right] \\
\equiv & \mathrm{A}_{m, k}^{n}+\sum_{i=0}^{n-1} \alpha_{i} \mathrm{~A}_{m+i r_{s}, k+i s}^{n-i} \\
& -\sum_{i=0}^{n-1} \alpha_{i} a_{r_{s}, s}^{(1)}\left(\delta_{0}\left(\mathrm{~A}_{r_{s}, s}^{0}\right)-\delta_{0}\left(\mathrm{~A}_{m+i r_{s}, k+i s}^{n-i-1}\right)\right) \lambda_{n-i, m+i r_{s}}^{0, r_{s}}(0) \mathrm{A}_{m+i r_{s}+r_{s}, k+i s+s}^{n-i-1} \\
= & \mathrm{A}_{m, k}^{n}+\alpha_{0} \mathrm{~A}_{m, k}^{n}+\sum_{i=1}^{n-1} \alpha_{i} \mathrm{~A}_{m+i r_{s}, k+i s}^{n-i} \\
& -\sum_{i=1}^{n-1} \alpha_{i-1} a_{r_{s}, s}^{(1)}\left(\delta_{0}\left(\mathrm{~A}_{r_{s}, s}^{0}\right)-\delta_{0}\left(\mathrm{~A}_{m+i r_{s}-r_{s}, k+i s-s}^{n-i}\right)\right) \lambda_{n-i+1, m+i r_{s}-r_{s}}^{0, r_{s}}(0) \mathrm{A}_{m+i r_{s}, k+i s}^{n-i} \\
& -\alpha_{n-1} a_{r_{s}, s}^{(1)}\left(\delta_{0}\left(\mathrm{~A}_{r_{s}, s}^{0}\right)-\delta_{0}\left(\mathrm{~A}_{m+n r_{s}-r_{s}, k+n s-s}^{0}\right)\right) \lambda_{1, m+n r_{s}-r_{s}}^{0, r_{s}}(0) \mathrm{A}_{m+n r_{s}, k+n s}^{0} .
\end{aligned}
$$

Now, let $\alpha_{0}=-1$ and

$\alpha_{i}-\alpha_{i-1} a_{r_{s}, s}^{(1)}\left(\delta_{0}\left(\mathrm{~A}_{r_{s}, s}^{0}\right)-\delta_{0}\left(\mathrm{~A}_{m+i r_{s}-r_{s}, k+i s-s}^{n-i+1}\right)\right) \lambda_{n-i+1, m+i r_{s}-r_{s}}^{0, r_{s}}(0)=0, \quad$ for $1 \leq i \leq n-1$.

Hence, we find

$$
\alpha_{i}=-\prod_{j=1}^{i} a_{r_{s}, s}^{(1)}\left(\delta_{0}\left(\mathrm{~A}_{r_{s}, s}^{0}\right)-\delta_{0}\left(\mathrm{~A}_{m+j r_{s}-r_{s}, k+j s-s}^{n-j+1}\right)\right) \lambda_{n-j+1, m+j r_{s}-r_{s}}^{0, r_{s}}(0), \quad \text { for } 0 \leq i \leq n-1
$$

Therefore,

$$
\left[\mathrm{T}_{m, k}^{n}, \mathrm{X}^{r_{s}+2 s}\right]+\mathrm{A}_{m, k}^{n}=-\alpha_{n} \mathrm{~A}_{m+n r_{s}, k+n s}^{0} .
$$

This is exactly the statement in the theorem. 
Theorem 5.8. There exists a sequence of invertible transformation that sends (5.13) into the second level normal form

$$
\mathrm{v}^{(2)}:=\mathrm{N}+a_{r_{s}, s}^{(1)} \mathrm{A}_{r_{s}, s}^{0}+\sum_{p=r_{s}+1}^{\infty} \sum_{q=s+1}^{\infty} a_{p, q}^{(2)} \mathrm{A}_{p, q}^{0},
$$

in which $a_{p, q}^{(2)}=0$ where $p \stackrel{\left(1+2 r_{s}\right)}{\equiv} r_{s}, q=k+(1+2 m) s$ and $m+2 k \neq r_{s}+2 s$, for natural numbers $m$.

Proof. From

$$
\left[\mathrm{N}, \mathrm{A}_{i, k}^{l}\right]=\mathrm{A}_{i, k}^{l+1}
$$

we observe that, the null space of $\mathrm{N}$ is generated by $\mathrm{A}_{m, k}^{2 m}$ for all natural numbers $m, k$. Therefore, we use this kernel term to remove an extra term from (5.13):

$$
\left[\mathrm{A}_{m, k}^{2 m}, \mathrm{X}^{r_{s}+2 s}\right] \equiv\left(\delta_{0}\left(\mathrm{~A}_{r_{s}, s}^{0}\right)-\delta_{0}\left(\mathrm{~A}_{m, k}^{2 m}\right)\right) \lambda_{0, r_{s}}^{2 m, m}(0) \mathrm{A}_{m+r_{s}, k+s}^{2 m} .
$$

Assume that $\delta_{0}\left(\mathrm{~A}_{r_{s}, s}^{0}\right) \neq \delta_{0}\left(\mathrm{~A}_{m, k}^{2 m}\right)$. Using Theorem (5.7) we know that there exists a transformation as $\mathrm{T}_{m+r_{s}, k+s}^{2 m}$ such that

$$
\begin{aligned}
& {\left[\mathrm{A}_{m, k}^{2 m}+\left(\delta_{0}\left(\mathrm{~A}_{r_{s}, s}^{0}\right)-\delta_{0}\left(\mathrm{~A}_{m, k}^{2 m}\right)\right) \lambda_{0, r_{s}}^{2 m, m}(0) \mathrm{T}_{m+r_{s}, k+s}^{2 m}, \mathrm{X}^{r_{s}+2 s}\right]} \\
& =-\left(\delta_{0}\left(\mathrm{~A}_{r_{s}, s}^{0}\right)-\delta_{0}\left(\mathrm{~A}_{m, k}^{2 m}\right)\right) \lambda_{0, r_{s}}^{2 m, m}(0) \alpha_{2 m} \mathrm{~A}_{m+r_{s}+2 m r_{s}, k+s+2 m s}^{0}
\end{aligned}
$$

which is non-zero. Hence, the proof follows.

As it shows for $\delta_{0}\left(\mathrm{~A}_{r_{s}, s}^{0}\right)=\delta_{0}\left(\mathrm{~A}_{m, k}^{2 m}\right)$ which reads $m_{1}=m=r_{s}+2(s-k)$ the term $\mathrm{A}_{m_{1}, k}^{2 m_{1}}$ is kernel of $\mathrm{X}^{r_{s}+2 s}$. We shall use this kernel for further possible reduction of terms form the (5.14). Rewrite (5.14) by

$$
\mathrm{v}^{(2)}:=\mathrm{N}+a_{r_{s}, s}^{(1)} \mathrm{A}_{r_{s}, s}^{0}+a_{r_{s_{1}}, s_{1}}^{(2)} \mathrm{A}_{r_{s_{1}}, s_{1}}^{0}+\sum_{p=r_{s_{1}}+1}^{\infty} \sum_{q=s_{1}+1}^{\infty} a_{p, q}^{(2)} \mathrm{A}_{p, q}^{0} .
$$

Theorem 5.9. The unique normal form of (5.13) is given by

$$
\mathrm{v}^{(3)}:=\mathrm{N}+a_{r_{s}, s}^{(1)} \mathrm{A}_{r_{s}, s}^{0}+a_{r_{s_{1}}, s_{1}}^{(2)} \mathrm{A}_{r_{s_{1}}, s_{1}}^{0}+\sum_{p=r_{s_{1}}+1}^{\infty} \sum_{p=s_{1}+1}^{\infty} a_{p, q}^{(3)} \mathrm{A}_{p, q}^{0},
$$

in which $a_{p, q}^{(3)}=0$ where $p \stackrel{\left(1+2 r_{s}\right)}{\equiv} r_{s}, p \stackrel{\left(1+2 r_{s_{1}}\right)}{\equiv} r_{s_{1}}$, and $q=k+s_{1}+2 m_{1} s_{1}$ where $m_{1} \in \mathbb{N}$ and $m_{1}=r_{s}+2(s-k)$.

Proof. The only transformation that has not been used so far is $\mathrm{A}_{m_{1}, k}^{2 m_{1}}$. Hereby,

$$
\left[\mathrm{A}_{m_{1}, k}^{2 m_{1}}, \mathrm{~A}_{r_{s_{1}}, s_{1}}^{0}\right] \equiv\left(\delta_{0}\left(\mathrm{~A}_{r_{s_{1}}, s_{1}}^{0}\right)-\delta_{0}\left(\mathrm{~A}_{m_{1}, k}^{2 m_{1}}\right)\right) \lambda_{0, r_{s}}^{2 m_{1}, m_{1}}(0) \mathrm{A}_{m_{1}+r_{s_{1}}, k+s_{1}}^{2 m_{1}}
$$

Using Theorem 5.7 we have

$$
\begin{aligned}
& {\left[\mathrm{A}_{m_{1}, k}^{2 m_{1}}, \mathrm{~A}_{r_{s_{1}}, s_{1}}^{0}\right]+\left[\left(\delta_{0}\left(\mathrm{~A}_{r_{s_{1}}, s_{1}}^{0}\right)-\delta_{0}\left(\mathrm{~A}_{m_{1}, k}^{2 m_{1}}\right)\right) \lambda_{0, r_{s}}^{2 m_{1}, m_{1}}(0) \mathrm{T}_{m_{1}+r_{s}, k+s}^{2 m_{1}}, \mathrm{X}^{r_{s}+2 s}\right] \equiv} \\
& -\alpha_{2 m_{1}}\left(\delta_{0}\left(\mathrm{~A}_{r_{s_{1}}, s_{1}}^{0}\right)-\delta_{0}\left(\mathrm{~A}_{m_{1}, k}^{2 m_{1}}\right)\right) \lambda_{0, r_{s}}^{2 m_{1}, m_{1}}(0) \mathrm{A}_{m_{1}+r_{s_{1}}+2 m_{1} r_{s}, k+s_{1}+2 m_{1} s_{1}}^{0} .
\end{aligned}
$$


Remark 5.10. One should note that for given $\delta=j$ one can have more than one kernel term. Then the matter is to figure out whether these kernel terms generate a common vector field in the process of finding the unique normal form. Assume that two elements with grade $j$ such as $\mathrm{A}_{m_{1}, k_{1}}^{2 m_{1}}, \mathrm{~A}_{m_{2}, k_{2}}^{2 m_{2}}$ where $m_{1} \neq m_{2}$ and $k_{1} \neq k_{2}$ are given. Then,

$$
\begin{aligned}
& {\left[\mathrm{A}_{m_{1}, k_{1}}^{2 m_{1}}, \mathrm{~A}_{r_{s}, s}^{0}\right]=\left\langle\mathrm{A}_{m_{1}+r_{s}-2 p_{1}, k_{1}+s+p_{1}}^{n_{1}}\right\rangle_{2 p_{1}+n_{1}=2 m_{1}}} \\
& {\left[\mathrm{~A}_{m_{2}, k_{2}}^{2 m_{2}}, \mathrm{~A}_{r_{s}, s}^{0}\right]=\left\langle\mathrm{A}_{m_{1}+r_{s}-2 p_{2}, k_{2}+s+p_{2}}^{n_{2}}\right\rangle_{2 p_{2}+n_{2}=2 m_{2}} .}
\end{aligned}
$$

Suppose that these kernel terms generate the same terms then it means that there exist $p_{1}$ and $p_{2}$ such that

$$
\mathrm{A}_{m_{1}+r_{s}-2 p_{1}, k_{1}+s+p_{1}}^{n_{1}}=\mathrm{A}_{m_{2}+r_{s}-2 p_{2}, k_{2}+s+p_{2}}^{n_{2}}
$$

and this implies that $n_{1}=n_{2}, m_{1}-2 p_{1}=m_{2}-2 p_{2}$, and $k_{1}+p_{1}=k_{2}+p_{2}$. On the other hand we have

$$
2 p_{1}+n_{1}=2 m_{1}, \text { and } 2 p_{2}+n_{2}=2 m_{2}
$$

since $n_{1}=n_{2}$ then one has $m_{1}-m_{2}=p_{1}-p_{2}$ which is in contradiction with $m_{1}-m_{2}=$ $2\left(p_{1}-p_{2}\right)$ when $m_{1} \neq m_{2}$.

\section{Example from $\widehat{\mathscr{A}_{2}}$}

In this part, the unique normal form up to fourth order for a given 3D nilpotent singularities is computed using the results from Sections 4 and 5.2. Consider the following dynamical system

$$
\begin{aligned}
\dot{x}= & \left(a_{1,0}^{0} z+2 a_{1,0}^{1} y+2 a_{1,0}^{2} x\right) x \\
& +\left(a_{2,0}^{0} z^{2}+4 a_{2,0}^{1} y z-a_{0,1}^{0} y^{2}+8 a_{2,0}^{2} y^{2}+\left(a_{0,1}^{0}+4 a_{2,0}^{2}\right) x z+24 a_{2,0}^{3} x y+24 a_{2,0}^{4} x^{2}\right) x \\
& +\left(a_{3,0}^{0} z^{3}+6 a_{3,0}^{1} y z^{2}+\left(-a_{1,1}^{0}+24 a_{3,0}^{2}\right) y^{2} z+\left(-2 a_{1,1}^{1}+48 a_{3,0}^{3}\right) y^{3}+\left(a_{1,1}^{0}+6 a_{3,0}^{2}\right) x z^{2}\right. \\
& +\left(2 a_{1,1}^{1}+72 a_{3,0}^{3}\right) x y z+\left(-2 a_{1,1}^{2}+288 a_{3,0}^{4}\right) x y^{2}+\left(2 a_{1,1}^{2}+72 a_{3,0}^{4}\right) x^{2} z \\
& \left.+720 a_{3,0}^{5} x^{2} y+720 a_{3,0}^{6} x^{3}\right) x \\
\dot{y}= & +\left(a_{1,0}^{0} z+2 a_{1,0}^{1} y+2 a_{1,0}^{2} x\right) y+\left(a_{2,0}^{0} z^{2}+4 a_{2,0}^{1} y z-a_{0,1}^{0} y^{2}+8 a_{2,0}^{2} y^{2}+a_{0,1}^{0} x z+4 a_{2,0}^{2} x z\right. \\
& \left.+24 a_{2,0}^{3} x y+24 a_{2,0}^{4} x^{2}\right) y+\left(a_{3,0}^{0} z^{3}+6 a_{3,0}^{1} y z^{2}-a_{1,1}^{0} y^{2} z+24 a_{3,0}^{2} y^{2} z-2 a_{1,1}^{1} y^{3}+48 a_{3,0}^{3} y^{3}\right. \\
& +a_{1,1}^{0} x z^{2}+6 a_{3,0}^{2} x z^{2}+2 a_{1,1}^{1} x y z+72 a_{3,0}^{3} x y z-2 a_{1,1}^{2} x y^{2}+288 a_{3,0}^{4} x y^{2}+2 a_{1,1}^{2} x^{2} z \\
& \left.+72 a_{3,0}^{4} x^{2} z+720 a_{3,0}^{5} x^{2} y+720 a_{3,0}^{6} x^{3}\right) y \\
\dot{z}= & 2 y+\left(a_{1,0}^{0} z+2 a_{1,0}^{1} y+2 a_{1,0}^{2} x\right) z \\
& +\left(a_{2,0}^{0} z^{2}+4 a_{2,0}^{1} y z-a_{0,1}^{0} y^{2}+8 a_{2,0}^{2} y^{2}+a_{0,1}^{0} x z+4 a_{2,0}^{2} x z+24 a_{2,0}^{3} x y+24 a_{2,0}^{4} x^{2}\right) z \\
& +\left(a_{3,0}^{0} z^{3}+6 a_{3,0}^{1} y z^{2}-\left(a_{1,1}^{0}+24 a_{3,0}^{2}\right) y^{2} z-2 a_{1,1}^{1} y^{3}+48 a_{3,0}^{3} y^{3}+\left(a_{1,1}^{0}+6 a_{3,0}^{2}\right) x z^{2}\right. \\
& \left.+\left(2 a_{1,1}^{1}+72 a_{3,0}^{3}\right) x y z-\left(2 a_{1,1}^{2}+288 a_{3,0}^{4}\right) x y^{2}+\left(2 a_{1,1}^{2}+72 a_{3,0}^{4}\right) x^{2} z+720 a_{3,0}^{5} x^{2} y+720 a_{3,0}^{6} x^{3}\right) z
\end{aligned}
$$


or equivalently

$$
v_{[4]}^{(0: 0)}:=\mathrm{N}+v_{1}^{(0: 0)}+v_{2}^{(0: 0)}+v_{3}^{(0: 0)},
$$

where the subindex [4] tells us we do not consider terms of grade 4 and higher, and

$$
\begin{aligned}
v_{1}^{(0: 0)}= & a_{1,0}^{0} \mathrm{~A}_{1,0}^{0}+a_{1,0}^{2} \mathrm{~A}_{1,0}^{2}+a_{1,0}^{1} \mathrm{~A}_{1,0}^{1}, \\
v_{2}^{(0: 0)}= & a_{0,1}^{0} \mathrm{~A}_{0,1}^{0}+a_{2,0}^{1} \mathrm{~A}_{2,0}^{1}+a_{2,0}^{0} \mathrm{~A}_{2,0}^{0}+a_{2,0}^{2} \mathrm{~A}_{2,0}^{2}+a_{2,0}^{3} \mathrm{~A}_{2,0}^{3}+a_{2,0}^{4} \mathrm{~A}_{2,0}^{4}, \\
v_{3}^{(0: 0)}= & a_{1,1}^{0} \mathrm{~A}_{1,1}^{0}+a_{3,0}^{0} \mathrm{~A}_{3,0}^{0}+a_{1,1}^{1} \mathrm{~A}_{1,1}^{1}+a_{3,0}^{1} \mathrm{~A}_{3,0}^{1}+a_{1,1}^{2} \mathrm{~A}_{1,1}^{2}+a_{3,0}^{2} \mathrm{~A}_{3,0}^{2} \\
& +a_{3,0}^{3} \mathrm{~A}_{3,0}^{3}+a_{3,0}^{4} \mathrm{~A}_{3,0}^{4}+a_{3,0}^{5} \mathrm{~A}_{3,0}^{5}+a_{3,0}^{6} \mathrm{~A}_{3,0}^{6},
\end{aligned}
$$

and the coefficients are real constants.

Theorem 6.1. The unique normal form of (6.1) up to order four is given by Equivalently,

$$
\begin{aligned}
\dot{x}= & a_{0,1}^{0} z x+a_{0,1}^{0}\left(x z-y^{2}\right) x+a_{2,0}^{0} z^{2} x \\
& +\left(a_{1,0}^{1} a_{1,1}^{0}+24 a_{2,0}^{1} a_{3,0}^{2}-6 a_{1,0}^{2} a_{3,0}^{2}-6 a_{2,0}^{2} a_{3,0}^{2}+4 a_{2,0}^{3} a_{3,0}^{2}+a_{1,1}^{0}\right) x z\left(x z-y^{2}\right) \\
& +a_{3,0}^{0}\left(a_{1,0}^{1}+a_{2,0}^{1}+1\right) x z^{3}, \\
\dot{y}= & x+a_{0,1}^{0} z y+a_{0,1}^{0}\left(x z-y^{2}\right) y+a_{2,0}^{0} z^{2} y \\
& +\left(a_{1,0}^{1} a_{1,1}^{0}+24 a_{2,0}^{1} a_{3,0}^{2}-6 a_{1,0}^{2} a_{3,0}^{2}-6 a_{2,0}^{2} a_{3,0}^{2}+4 a_{2,0}^{3} a_{3,0}^{2}+a_{1,1}^{0}\right) y z\left(x z-y^{2}\right) \\
& a_{3,0}^{0}\left(a_{1,0}^{1}+a_{2,0}^{1}+1\right) y z^{3}, \\
\dot{z}= & 2 y+a_{0,1}^{0} z^{2}+a_{0,1}^{0}\left(x z-y^{2}\right) z+a_{2,0}^{0} z^{3} \\
& +\left(a_{1,0}^{1} a_{1,1}^{0}+24 a_{2,0}^{1} a_{3,0}^{2}-6 a_{1,0}^{2} a_{3,0}^{2}-6 a_{2,0}^{2} a_{3,0}^{2}+4 a_{2,0}^{3} a_{3,0}^{2}+a_{1,1}^{0}\right) z^{2}\left(x z-y^{2}\right), \\
& +a_{3,0}^{0}\left(a_{1,0}^{1}+a_{2,0}^{1}+1\right) z^{4} .
\end{aligned}
$$

And in terms of invariants $\mathfrak{a}, \mathfrak{b}$ is as

$$
\begin{aligned}
\dot{x}= & a_{0,1}^{0} \mathfrak{a} x+a_{0,1}^{0} \mathfrak{b} x+a_{2,0}^{0} \mathfrak{a}^{2} x \\
& +\left(a_{1,0}^{1} a_{1,1}^{0}+24 a_{2,0}^{1} a_{3,0}^{2}-6 a_{1,0}^{2} a_{3,0}^{2}-6 a_{2,0}^{2} a_{3,0}^{2}+4 a_{2,0}^{3} a_{3,0}^{2}+a_{1,1}^{0}\right) \mathfrak{a} \mathfrak{b} x \\
& +a_{3,0}^{0}\left(a_{1,0}^{1}+a_{2,0}^{1}+1\right) \mathfrak{a}^{3} x \\
\dot{y}= & x+a_{0,1}^{0} \mathfrak{a} y+a_{0,1}^{0} \mathfrak{b} y+a_{2,0}^{0} \mathfrak{a}^{2} y \\
& +\left(a_{1,0}^{1} a_{1,1}^{0}+24 a_{2,0}^{1} a_{3,0}^{2}-6 a_{1,0}^{2} a_{3,0}^{2}-6 a_{2,0}^{2} a_{3,0}^{2}+4 a_{2,0}^{3} a_{3,0}^{2}+a_{1,1}^{0}\right) \mathfrak{a} \mathfrak{b} y \\
& a_{3,0}^{0}\left(a_{1,0}^{1}+a_{2,0}^{1}+1\right) \mathfrak{a}^{3} y \\
\dot{z}= & 2 y+a_{0,1}^{0} \mathfrak{a} z+a_{0,1}^{0} \mathfrak{b} z+a_{2,0}^{0} \mathfrak{a}^{2} z \\
& +\left(a_{1,0}^{1} a_{1,1}^{0}+24 a_{2,0}^{1} a_{3,0}^{2}-6 a_{1,0}^{2} a_{3,0}^{2}-6 a_{2,0}^{2} a_{3,0}^{2}+4 a_{2,0}^{3} a_{3,0}^{2}+a_{1,1}^{0}\right) \mathfrak{a} \mathfrak{b} z \\
& +a_{3,0}^{0}\left(a_{1,0}^{1}+a_{2,0}^{1}+1\right) \mathfrak{a}^{3} z .
\end{aligned}
$$

Proof. At the first level of normal form we transform the first and the second order terms in the normal form simultaneously. Set

$$
\begin{aligned}
& \mathrm{T}_{1}^{1}=a_{1,0}^{1} \mathrm{~A}_{1,0}^{0}+a_{1,0}^{2} \mathrm{~A}_{1,0}^{1}, \\
& \mathrm{~T}_{2}^{1}=a_{2,0}^{1} \mathrm{~A}_{2,0}^{0}+a_{2,0}^{2} \mathrm{~A}_{2,0}^{1}+a_{2,0}^{3} \mathrm{~A}_{2,0}^{2}+a_{2,0}^{4} \mathrm{~A}_{2,0}^{3} .
\end{aligned}
$$


By applying this transformation we have

$$
\begin{aligned}
v_{[4]}^{(0: 1)} & :=v^{(0: 0)}+\left[\mathrm{T}_{1}^{1}+\mathrm{T}_{2}^{1}, v_{[4]}^{(0: 0)}\right] \\
& =\mathrm{N}+v_{1}^{(0: 1)}+v_{2}^{(0: 1)}+v_{3}^{(0: 1)} .
\end{aligned}
$$

In what follows, we compute a few structure constants as an illustration of Theorem 4.2.

For instance the Lie bracket of the first term of $\mathrm{T}_{1}^{1}$ with the last term of $v_{2}^{(0: 0)}$ contains

$$
\begin{aligned}
{\left[\mathrm{A}_{1,0}^{0}, \mathrm{~A}_{2,0}^{4}\right] } & =\sum_{\rho=0}^{1} \lambda_{4,2}^{0,1}(\rho) \mathrm{A}_{3-2 \rho, \rho}^{4-2 \rho} \\
& =\lambda_{4,2}^{0,1}(0) \mathrm{A}_{3,0}^{4}+\lambda_{4,2}^{0,1}(1) \mathrm{A}_{1,1}^{2} \\
& =\frac{48}{5} \mathrm{~A}_{3,0}^{4}+\frac{1}{15} \mathrm{~A}_{1,1}^{2} .
\end{aligned}
$$

Following (3.1) we have

$$
\begin{aligned}
{\left[A_{1,0}^{0}, A_{0,1}^{0}\right] } & =\lambda_{0,0}^{0,1}(0) A_{1,1}^{0}=A_{1,1}^{0} \\
{\left[A_{1,0}^{0}, A_{2,0}^{1}\right] } & =\lambda_{1,2}^{0,1}(0) A_{3,0}^{1}=\frac{2}{3} A_{3,0}^{1} \\
{\left[A_{1,0}^{0}, A_{2,0}^{0}\right] } & =\lambda_{0,3}^{0,1}(0) A_{3,0}^{0}=A_{3,0}^{0} \\
{\left[A_{1,0}^{0}, A_{2,0}^{2}\right] } & =\lambda_{2,2}^{0,1}(0) A_{3,0}^{2}+\lambda_{2,2}^{0,1}(1) A_{1,1}^{0}=\frac{2}{5} A_{3,0}^{2}+\frac{8}{5} A_{1,1}^{0} \\
{\left[A_{1,0}^{0}, A_{2,0}^{3}\right] } & =\lambda_{3,2}^{0,1}(0) A_{3,0}^{3}+\lambda_{3,2}^{0,1}(1) A_{1,1}^{1}=\frac{1}{5} A_{3,0}^{2}+\frac{24}{5} A_{1,1}^{0} .
\end{aligned}
$$

Note that for computing $\lambda_{l_{2}, \mu_{2}}^{l_{1}, \mu_{1}}(0)$ and $\lambda_{l_{2}, \mu_{2}}^{0, \mu_{1}}(\rho)$, one can use the explicit formulas of Remarks 3.6 and 3.4, respectively.

Now, we continue the normalization process. Hence, $v_{[4]}^{(0: 1)}$ has the following components

$$
\begin{aligned}
v_{1}^{(0: 1)}= & a_{1,0}^{0} \mathrm{~A}_{1,0}^{0}, \\
v_{2}^{(0: 1)}= & a_{0,1}^{0} \mathrm{~A}_{0,1}^{0}+a_{2,0}^{0} \mathrm{~A}_{2,0}^{0} \\
v_{3}^{(0: 1)}= & \left(a_{1,0}^{1} a_{1,1}^{0}+24 a_{2,0}^{1} a_{3,0}^{2}-6 a_{1,0}^{2} a_{3,0}^{2}-6 a_{2,0}^{2} a_{3,0}^{2}+4 a_{2,0}^{3} a_{3,0}^{2}+a_{1,1}^{0}\right) \mathrm{A}_{1,1}^{0}+a_{3,0}^{0}\left(a_{1,0}^{1}+a_{2,0}^{1}+1\right) \mathrm{A}_{3,0}^{0} \\
& +\left(24 a_{1,0}^{1} a_{3,0}^{3}+a_{1,0}^{2} a_{1,1}^{1}+24 a_{2,0}^{2} a_{3,0}^{3}-16 a_{2,0}^{3} a_{3,0}^{3}+24 a_{4,2,0} a_{3,0}^{3}+a_{1,1}^{1}\right) \mathrm{A}_{1,1}^{1} \\
& +a_{3,0}^{1}\left(a_{1,0}^{1}+a_{2,0}^{1}+a_{1,0}^{2}+a_{2,0}^{2}+1\right) \mathrm{A}_{3,0}^{1} \\
& +\left(144 a_{1,0}^{1} a_{3,0}^{4}-36 a_{1,0}^{2} a_{3,0}^{4}+24 a_{2,0}^{3} a_{3,0}^{4}-36 a_{2,0}^{4} a_{3,0}^{4}+a_{1,1}^{2}\right) \mathrm{A}_{1,1}^{2} \\
& +\left(a_{1,0}^{1} a_{3,0}^{2}+a_{2,0}^{1} a_{3,0}^{2}+a_{1,0}^{2} a_{3,0}^{2}+a_{2,0}^{2} a_{3,0}^{2}+a_{2,0}^{3} a_{3,0}^{2}+a_{3,0}^{2}\right) \mathrm{A}_{3,0}^{2} \\
& +\left(a_{1,0}^{1} a_{3,0}^{3}+a_{1,0}^{2} a_{3,0}^{3}+a_{2,0}^{2} a_{3,0}^{3}+a_{2,0}^{3} a_{3,0}^{3}+a_{2,0}^{4} a_{3,0}^{3}+a_{3,0}^{3}\right) \mathrm{A}_{3,0}^{3} \\
& +\left(a_{1,0}^{1} a_{3,0}^{4}+a_{1,0}^{2} a_{3,0}^{4}+a_{2,0}^{3} a_{3,0}^{4}+a_{2,0}^{4} a_{3,0}^{4}+a_{3,0}^{4}\right) \mathrm{A}_{3,0}^{4}+\left(a_{1,0}^{2} a_{3,0}^{5}+a_{2,0}^{4} a_{3,0}^{5}+a_{3,0}^{5}\right) \mathrm{A}_{3,0}^{5} \\
& +a_{3,0}^{6} \mathrm{~A}_{3,0}^{6} .
\end{aligned}
$$


At the end we apply $\mathrm{T}_{3}^{2}$ to $v_{[4]}^{(0: 1)}$ to find $v_{[4]}^{(0: 2)}$. Define

$$
\begin{aligned}
\mathrm{T}_{3}^{2}:= & \left(24 a_{1,0}^{1} a_{3,0}^{3}+a_{1,0}^{2} a_{1,1}^{1}+24 a_{2,0}^{2} a_{3,0}^{3}-16 a_{2,0}^{3} a_{3,0}^{3}+24 a_{2,0}^{4} a_{3,0}^{3}+a_{1,1}^{1}\right) \mathrm{A}_{1,1}^{0} \\
& +a_{3,0}^{1}\left(a_{1,0}^{1}+a_{2,0}^{1}+a_{1,0}^{2}+a_{2,0}^{2}+1\right) \mathrm{A}_{3,0}^{0} \\
& +\left(144 a_{1,0}^{1} a_{3,0}^{4}-36 a_{1,0}^{2} a_{3,0}^{4}+24 a_{2,0}^{3} a_{3,0}^{4}-36 a_{2,0}^{4} a_{3,0}^{4}+a_{1,1}^{2}\right) \mathrm{A}_{1,1}^{1} \\
& +\left(a_{1,0}^{1} a_{3,0}^{2}+a_{2,0}^{1} a_{3,0}^{2}+a_{1,0}^{2} a_{3,0}^{2}+a_{2,0}^{2} a_{3,0}^{2}+a_{2,0}^{3} a_{3,0}^{2}+a_{3,0}^{2}\right) \mathrm{A}_{3,0}^{1} \\
& +\left(a_{1,0}^{1} a_{3,0}^{3}+a_{1,0}^{2} a_{3,0}^{3}+a_{2,0}^{2} a_{3,0}^{3}+a_{2,0}^{3} a_{3,0}^{3}+a_{2,0}^{4} a_{3,0}^{3}+a_{3,0}^{3}\right) \mathrm{A}_{3,0}^{2} \\
& +\left(a_{1,0}^{1} a_{3,0}^{4}+a_{1,0}^{2} a_{3,0}^{4}+a_{2,0}^{3} a_{3,0}^{4}+a_{2,0}^{4} a_{3,0}^{4}+a_{3,0}^{4}\right) \mathrm{A}_{3,0}^{3} \\
& +\left(a_{1,0}^{2} a_{3,0}^{5}+a_{2,0}^{4} a_{3,0}^{5}+a_{3,0}^{5}\right) \mathrm{A}_{3,0}^{4}+a_{3,0}^{6} \mathrm{~A}_{3,0}^{5} .
\end{aligned}
$$

Then, we have

$$
\begin{aligned}
v_{[4]}^{(0: 2)} & :=v^{(0: 1)}+\left[\mathrm{T}_{3}^{2}, v^{(0: 1)}\right] \\
& =\mathrm{N}+v_{1}^{(0: 1)}+v_{2}^{(0: 1)}+v_{3}^{(0: 2)}
\end{aligned}
$$

where

$$
\begin{aligned}
v_{3}^{(0: 2):=} & \left(a_{1,0}^{1} a_{1,1}^{0}+24 a_{2,0}^{1} a_{3,0}^{2}-6 a_{1,0}^{2} a_{3,0}^{2}-6 a_{2,0}^{2} a_{3,0}^{2}+4 a_{2,0}^{3} a_{3,0}^{2}+a_{1,1}^{0}\right) \mathrm{A}_{1,1}^{0} \\
& +a_{3,0}^{0}\left(a_{1,0}^{1}+a_{2,0}^{1}+1\right) \mathrm{A}_{3,0}^{0} .
\end{aligned}
$$

Following Section 5.2 the ker ad $\mathrm{N}_{\mathrm{N}}$-terms can not eliminate any terms from $v_{[4]}^{(0: 2)}$, since the grade is always less than seven. Hence, the second level normal form $v_{[4]}^{(0: 2)}$ is the unique normal form (up till grade three).

\section{Concluding remarks}

We have shown how the inversion formula for the Clebsch-Gordan coefficients (or $3 j$-symbols) enables us to determine the structure constants for a basis of the Lie algebra of polynomial vector fields that is adapted to the action of the nilpotent linear vector field in the $3 \mathrm{D}$ case. In principle, this is a procedure that could be generalized to higher dimensions, although this will no doubt require some serious work.

Once the structure constants have been determined, the analysis of the higher level normal form, and ultimately the unique normal form, comes within reach. Even in the 2D case, this is a nontrivial problem, that will have more and more subcases as the dimension grows.

In addition, these formulas may prove useful in the study of the convergence of the (first level) normal form where one needs to estimate the transformation through the Homological Equation. We refer the interested reader to [15], where parts of the proof show a striking similarity to the techniques employed in the present paper and we hope to improve on these results using the techniques described in [11].

\section{References}

[1] Alberto Baider and Jan A Sanders. Unique normal forms: The nilpotent Hamiltonian case. Journal of differential equations, 92(2):282-304, 1991. 
[2] Alberto Baider and Jan A Sanders. Further reduction of the Takens-Bogdanov normal form. Journal of Differential Equations, 99(2):205-244, 1992.

[3] David H Collingwood and William M McGovern. Nilpotent orbits in semisimple Lie algebra: an introduction. CRC Press, 1993.

[4] Robert W. Donley, Jr. and Won Geun Kim. A rational theory of Clebsch-Gordan coefficients. In Representation theory and harmonic analysis on symmetric spaces, volume 714 of Contemp. Math., pages 115-130. Amer. Math. Soc., Providence, RI, 2018.

[5] Igor Frenkel, Catharina Stroppel, and Joshua Sussan. Categorifying fractional Euler characteristics, Jones-Wenzl projector and 3j-symbols. Quantum Topology, 3:181-253, 2012.

[6] Majid Gazor, Fahimeh Mokhtari, and Jan A Sanders. Vector potential normal form classification for completely integrable solenoidal nilpotent singularities. Journal of Differential Equations, 267:3083-3113, 2019.

[7] Christian Kassel. Quantum groups, volume 155. Springer Science \& Business Media, 2012.

[8] Anatolii Nikolaevich Kirillov. The quantum Clebsch-Gordan coefficients. Zapiski Nauchnykh Seminarov POMI, 168:67-84, 1988.

[9] Anthony W. Knapp. Lie groups beyond an introduction, volume 140. Springer Science \& Business Media, 2013.

[10] Tom H Koornwinder. Clebsch-Gordan coefficients for SU(2) and Hahn polynomials. Nieuw Archief voor de Wiskunde (3), XXIX:140-155, 1981.

[11] Fahimeh Mokhtari and Jan A Sanders. Versal normal form for nonsemisimple singularities. Journal of Differential Equations, 267(5):3083-3113, 2019.

[12] Peter J Olver. Classical invariant theory, volume 44. Cambridge University Press, 1999.

[13] Jan A Sanders. Normal form theory and spectral sequences. Journal of Differential Equations, 192(2):536-552, 2003.

[14] Jan A Sanders, F. Verhulst, and J. Murdock. Averaging methods in nonlinear dynamical systems, volume 59 of Applied Mathematical Sciences. Springer, New York, second edition, 2007.

[15] Laurent Stolovitch and Freek Verstringe. Holomorphic normal form of nonlinear perturbations of nilpotent vector fields. Regular and Chaotic Dynamics, 21(4):410-436, 2016.

[16] Bartel L van der Waerden. Group theory and quantum mechanics, volume 214. Springer Science \& Business Media, 2012. 